\title{
Kinetic Resolution of Racemic 2-Hydroxyamides Using a Diphenylacetyl Component as an Acyl Source and a Chiral Acyl-Transfer Catalyst
}

\author{
Takatsugu Murata ${ }^{1}$, Tatsuya Kawanishi ${ }^{1}$, Akihiro Sekiguchi ${ }^{1}$, Ryo Ishikawa ${ }^{1}$, Keisuke Ono ${ }^{1}$,

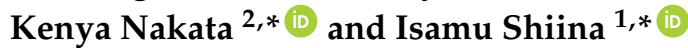 \\ 1 Department of Appliaed Chemistry, Faculty of Science, Tokyo University of Science, Tokyo 162-8601, Japan; \\ b116707@ed.tus.ac.jp (T.M.); tatsuya.kawanishi@tcichemicals.com (T.K.); \\ akihiro.sekiguchi@nipponkayaku.co.jp (A.S.); 12ishikawa25@gmail.com (R.I.); ki_ono@jp.daicel.com (K.O.) \\ 2 Graduate School of Natural Science and Technology, Shimane University, Shimane 690-8504, Japan \\ * Correspondence: nakata@riko.shimane-u.ac.jp (K.N.); shiina@rs.kagu.tus.ac.jp (I.S.); \\ Tel.: +81-852-32-6424 (K.N.); +81-3-3260-4271 (I.S.)
}

Received: 23 July 2018; Accepted: 7 August 2018; Published: 10 August 2018

\begin{abstract}
Various optically active 2-hydroxyamide derivatives are produced based on the kinetic resolution of racemic 2-hydroxyamides with a diphenylacetyl component and $(R)$-benzotetramisole $((R)-B T M)$, a chiral acyl-transfer catalyst, via asymmetric esterification and acylation. It was revealed that a tertiary amide can be used with this novel protocol to achieve high selectivity (22 examples; $s$-value reaching over 250). The resulting chiral compounds could be transformed into other useful structures while maintaining their chirality.
\end{abstract}

Keywords: kinetic resolution; 2-hydroxyamide; organocatalysis; Weinreb amide; esterification; carboxylic anhydride

\section{Introduction}

Optically active 2-hydroxyamide derivatives are frequently utilized as chiral building blocks not only for synthesizing biologically active compounds [1-4], but also for preparing asymmetric catalysts and chiral auxiliaries [5,6]. Consequently, considerable effort has been devoted toward developing efficient methods for synthesizing these compounds, including enzymatic [7] and chemical transformations [8-10]. For the purpose of providing chiral alcohols, the kinetic resolution (KR) of racemic alcohols by asymmetric acylation using organocatalysis is widely used as one of the most effective methods [11-13]. However, to the best of our knowledge, a general method for the kinetic resolution of racemic 2-hydroxyamide derivatives has not been reported to date. We recently accomplished the first KR of racemic alcohols with achiral carboxylic acids and of racemic carboxylic acids with achiral alcohols by asymmetric esterification [14-27] via the in situ formation of a mixed anhydride using carboxylic anhydrides as coupling reagents combined with chiral acyl-transfer catalysts. Furthermore, KR of racemic 2-hydroxyalkanoates with diphenylacetic acid was achieved using pivalic anhydride in the presence of $(R)$-benzotetramisole $[27,28]((R)$-BTM; Scheme 1; (i)). Therefore, it was hypothesized that this KR protocol could be similarly applied to 2-hydroxyamide derivatives (Scheme 1; (ii)). In this article, we report the novel KR of various racemic 2-hydroxyamide derivatives using a diphenylacetyl component as an acyl source, catalyzed by $(R)-\mathrm{BTM}$. 

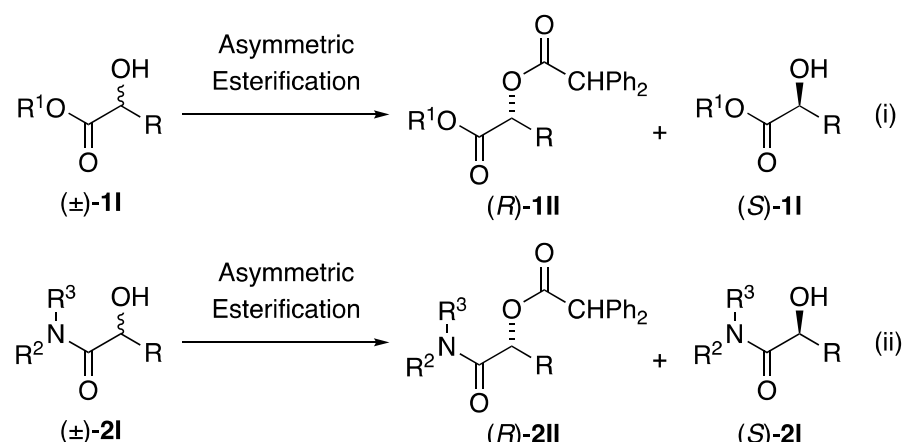

Scheme 1. Our previous result (i) and working hypothesis for the present study (ii).

\section{Results and Discussion}

To determine suitable structures for the amide moiety, the KR reactions of a variety of racemic 2-hydroxyamides were initially examined using diphenylacetyl sources derived from $\mathrm{Ph}_{2} \mathrm{CHCO}_{2} \mathrm{H}$ or $\left(\mathrm{Ph}_{2} \mathrm{CHCO}\right)_{2} \mathrm{O}$ (DPHAA) [29], catalyzed by (R)-BTM in $\mathrm{Et}_{2} \mathrm{O}$ at room temperature for $12 \mathrm{~h}$, which were reaction conditions similar to those established in the previous study (Table 1). We first performed the KR of the secondary $N$-alkyl amide with methyl $( \pm)-\mathbf{1 a}$ or benzyl $( \pm)-\mathbf{1 b}$ and $N$-phenyl amide $( \pm)-\mathbf{1 c}$ via asymmetric esterification. These substrates were found to be unsuitable for the reaction (Entries 1-3). Conversely, it was found that the tertiary amide yielded high s-values under the reaction conditions [30]. The KR of ( \pm )-1d smoothly proceeded, affording the corresponding ester $(R)$-2d $(48 \% ; 92 \%$ ee) and the recovered alcohol $(S)-1 \mathbf{d}(46 \% ;>99 \%$ ee) with a high $s$-value (Entry $4 ; s=254)$. It is noteworthy that $N$-methoxy- $N$-methylamide ( \pm )-1e (known as Weinreb amide) [31-33] was successfully applied to this protocol with high synthetic utility (Entry $5 ; s=156$ ). As the tertiary amide was recognized as a suitable structure for attaining high selectivity, we subsequently performed the $\mathrm{KR}$ via asymmetric acylation and not via asymmetric esterification for the same reaction. As expected, high selectivity was also achieved by the reaction of $( \pm)-\mathbf{1} \mathbf{d}$ and $\mathbf{1 e}$ using the asymmetric acylation protocol (Entries 6 and 7).

Table 1. Kinetic resolution (KR) of various racemic 2-hydroxyamides (( \pm$)$-1a-1e).

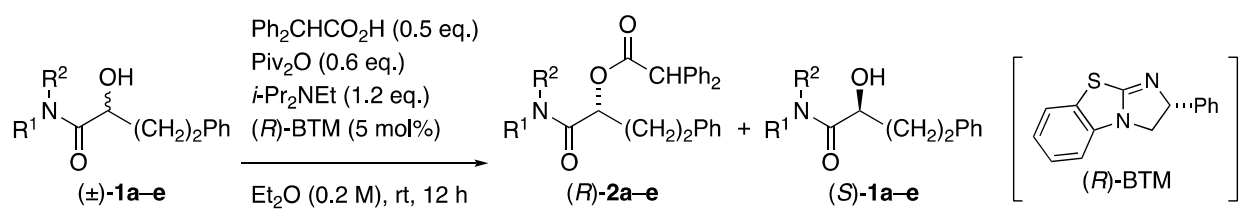

\begin{tabular}{|c|c|c|c|c|}
\hline Entry & $\mathbf{R}^{1}, \mathbf{R}^{2}$ & Yield $(2 ; 1)[\%]$ & ee $(2 ; 1)[\%]$ & $s$ \\
\hline 1 & $\mathrm{Me}, \mathrm{H}(\mathbf{a})$ & $42 ; 52$ & $12 ; 14$ & 1 \\
\hline 2 & $\mathrm{Bn}, \mathrm{H}(\mathbf{b})$ & $50 ; 50$ & $17 ; 15$ & 2 \\
\hline 3 & $\mathrm{Ph}, \mathrm{H}(\mathrm{c})$ & $55 ; 45$ & $79 ; 73$ & 18 \\
\hline $4^{\mathrm{a}}$ & $\mathrm{Me}, \mathrm{Me}(\mathbf{d})$ & $48 ; 46$ & $92 ;>99$ & 254 \\
\hline $5^{a}$ & $\mathrm{Me}, \mathrm{OMe}(\mathbf{e})$ & $50 ; 50$ & $94 ; 99$ & 156 \\
\hline $6^{b}$ & $\mathrm{Me}, \mathrm{Me}(\mathbf{f})$ & $47 ; 50$ & $91 ; 98$ & 95 \\
\hline $7^{c}$ & $\mathrm{Me}, \mathrm{OMe}(\mathrm{g})$ & $47 ; 42$ & $94 ; 98$ & 157 \\
\hline
\end{tabular}

a $\mathrm{Ph}_{2} \mathrm{CHCO}_{2} \mathrm{H}$ (0.75 eq.), $\mathrm{Piv}_{2} \mathrm{O}$ (0.9 eq.), and $i$ - $\mathrm{Pr}_{2} \mathrm{NEt}\left(1.2\right.$ eq.) were used. ${ }^{\mathrm{b}}\left(\mathrm{Ph}_{2} \mathrm{CHCO}\right)_{2} \mathrm{O}$ (DPHAA; 0.60 eq.) and $i$ - $\mathrm{Pr}_{2} \mathrm{NEt}\left(0.60\right.$ eq.) were used. ${ }^{c}\left(\mathrm{Ph}_{2} \mathrm{CHCO}\right)_{2} \mathrm{O}$ (DPHAA; 0.75 eq.) and $i-\mathrm{Pr}_{2} \mathrm{NEt}$ ( 0.75 eq.) were used.

To assess the generality of this novel method, various racemic 2-hydroxy- $N, N$-dimethylamides $( \pm)$-3a-3k with different substituted forms (Table 2) were subjected to asymmetric esterification (condition A1) and asymmetric acylation (condition B1). When the KR of $\mathbf{3 a}-\mathbf{3 c}$, $\mathbf{3 e}$, and $\mathbf{3 h}$, bearing normal aliphatic alkyl chains at the C-2 positions, was performed under the conditions $\mathrm{A} 1$ and $\mathrm{B} 1$, the reaction successfully proceeded with high s-values in all cases. Asymmetric esterification (condition A1) tended to show better results than asymmetric acylation (condition B1); however, it was revealed 
that the chiral acylation protocol was also useful for obtaining good s-values. In contrast, the reaction of $( \pm)-\mathbf{3 d}$ and $\mathbf{3 g}$, bearing branched aliphatic alkyl chains $(\mathrm{R}=i$-Pr and $c-H e x)$ at the $C-2$ positions, showed a slight decrease in selectivity, while the reaction of $3 f(R=i$-Bu) yielded a good $s$-value. We also examined several racemic $\omega$-(tert-butyldimethylsiloxy)-2-hydroxy- $N, N$-dimethylamide derivatives $( \pm)-3 \mathbf{i}-3 \mathbf{k}$, having different methylene lengths, as shown in Entries 17-22. It was found that the selectivity of the KR of $( \pm)-3 \mathbf{i}$ was somewhat lowered by the influence of the siloxy group at the C-3 position (Entries 17 and 18). Other reactions yielded high s-values, regardless of the length of the alkyl chains possessing tert-butyldimethylsiloxy groups under the conditions A1 and B1 (Entries 19-22).

Table 2. KR of 2-hydroxy dimethylamide (( \pm -3a-3k).

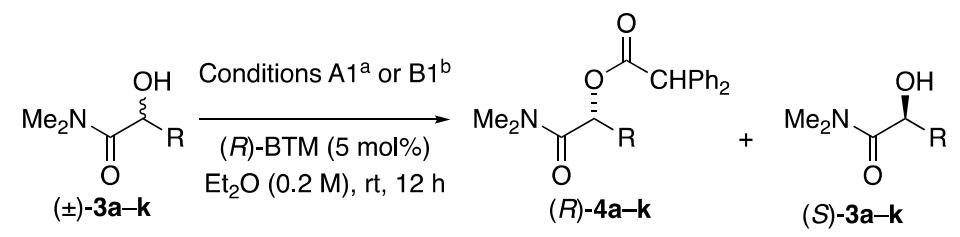

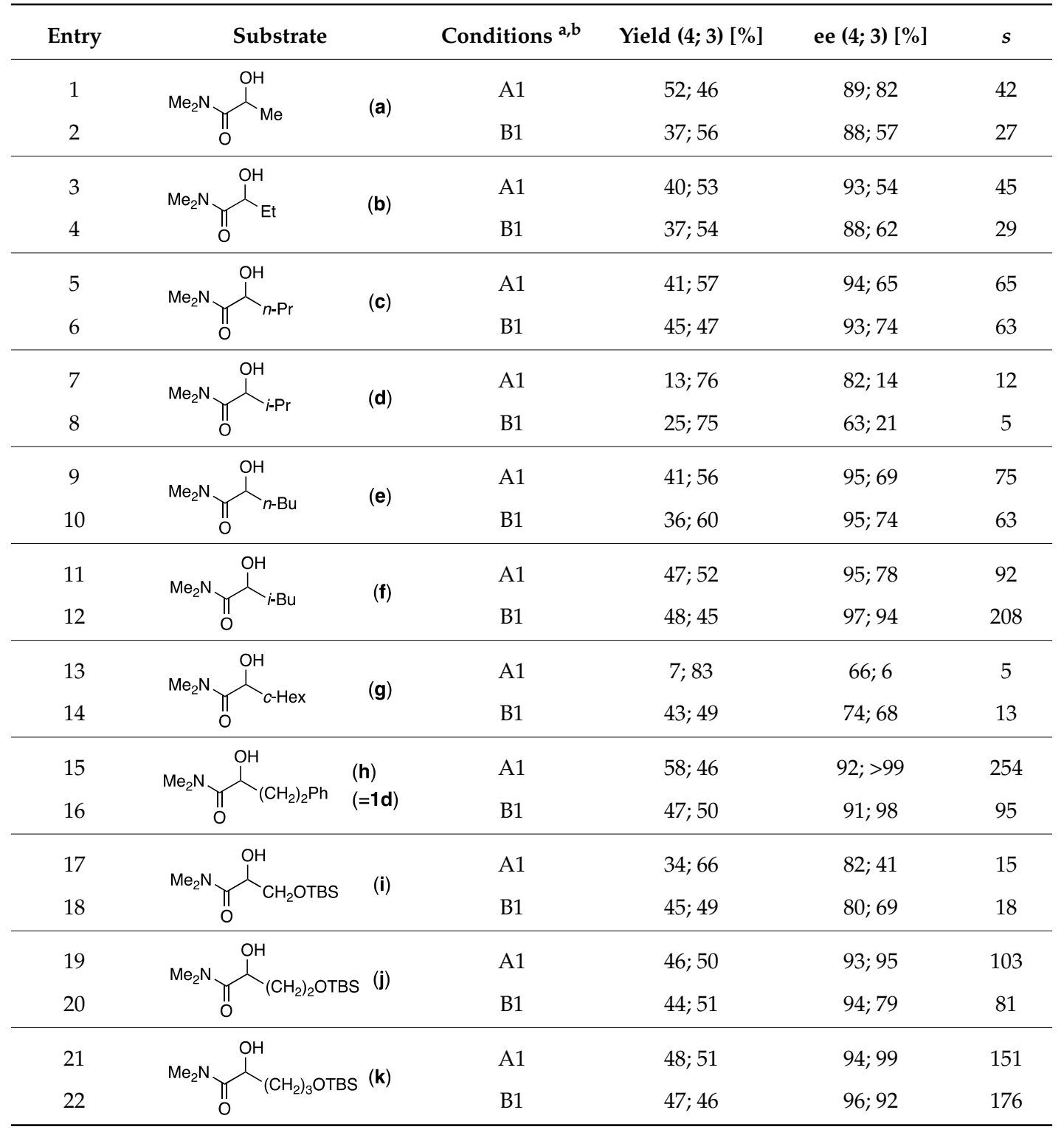

a Conditions $\mathrm{A} 1 ; \mathrm{Ph}_{2} \mathrm{CHCO}_{2} \mathrm{H}$ (0.75 eq.), $\mathrm{Piv}_{2} \mathrm{O}$ (0.9 eq.), and $i-\mathrm{Pr}_{2} \mathrm{NEt}\left(1.2\right.$ eq.). ${ }^{\mathrm{b}}$ Conditions $\mathrm{B} 1 ;\left(\mathrm{Ph}_{2} \mathrm{CHCO}\right)_{2} \mathrm{O}$ (DPHAA; 0.60 eq.) and $i-\operatorname{Pr}_{2} \mathrm{NEt}(0.60$ eq.). 
Furthermore, we performed the KR of various racemic 2-hydroxy-Weinreb amides $( \pm)$-5a-5k with substitution patterns corresponding to the $N, N$-dimethylamides $( \pm)$-3a-3k using a similar protocol (Table 3). Consequently, the same tendency was observed. The KR of 2-hydroxy-Weinreb amides 5a-5c, $\mathbf{5 e}, \mathbf{5 f}, \mathbf{5 h}, \mathbf{5} \mathbf{j}$, and $\mathbf{5 k}$, bearing normal aliphatic alkyl chains at the C-2 positions, exhibited high s-values in all cases under the conditions A1 and B2. Conversely, the reactions of 2-hydroxy-Weinreb amides $( \pm)-5 d, 5 g$, and $\mathbf{5 i}$, bearing branched aliphatic alkyl chains at the C-2 positions or a siloxy group at the C-3 position, exhibited decreased selectivity.

Table 3. KR of 2-hydroxy-Weinreb amide (( \pm$)-5 \mathbf{a}-5 \mathbf{k})$.

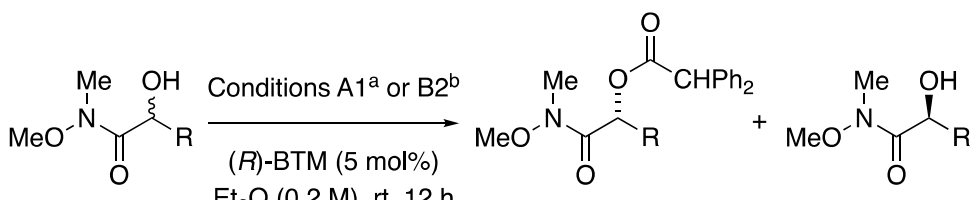

$( \pm)-5 \mathbf{a}-\mathbf{k} \quad \mathrm{Et}_{2} \mathrm{O}(0.2 \mathrm{M}), \mathrm{rt}, 12 \mathrm{~h}$

(R)-6a-k

(S) $-\mathbf{5 a}-\mathbf{k}$

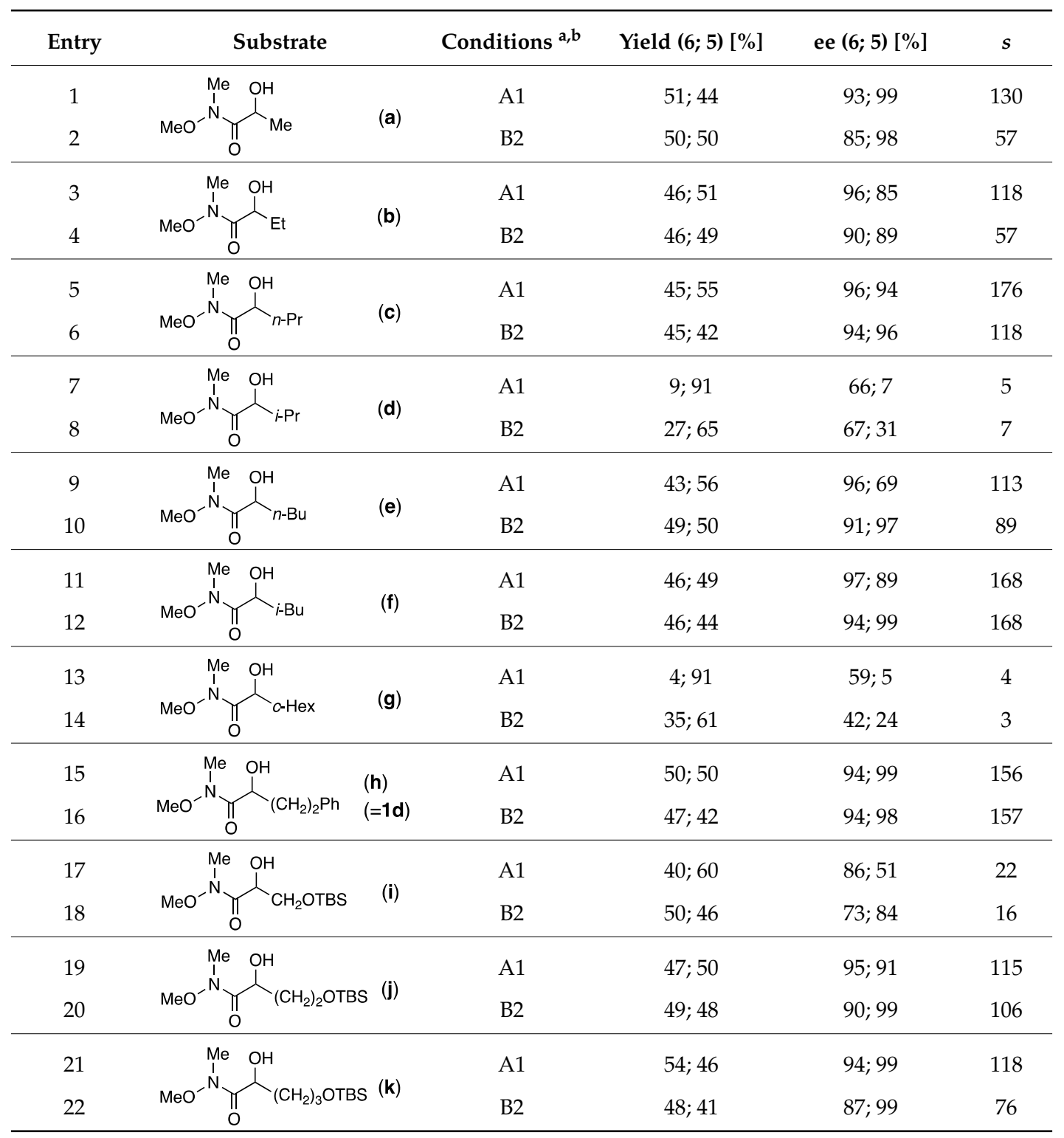

a Conditions $\mathrm{A} 1 ; \mathrm{Ph}_{2} \mathrm{CHCO}_{2} \mathrm{H}$ (0.75 eq.), $\mathrm{Piv}_{2} \mathrm{O}$ (0.9 eq.), and $i-\mathrm{Pr}_{2} \mathrm{NEt}$ (1.2 eq.). ${ }^{\mathrm{b}}$ Conditions $\mathrm{B} 2 ;\left(\mathrm{Ph}_{2} \mathrm{CHCO}\right)_{2} \mathrm{O}$ (DPHAA; 0.75 eq.) and $i-\mathrm{Pr}_{2} \mathrm{NEt}$ (0.75 eq.). 
To support the results of the experimental data, we calculated the transition state of each enantiomer in the KR. This was performed using density functional theory (DFT) calculations at the B3LYP / 6-31G*//B3LYP / 6-31G* level according to a previously reported method $[23,27,28]$. Initially, we conducted a theoretical study on the KR of 2-hydroxy dimethylamides (Scheme 2) [34].

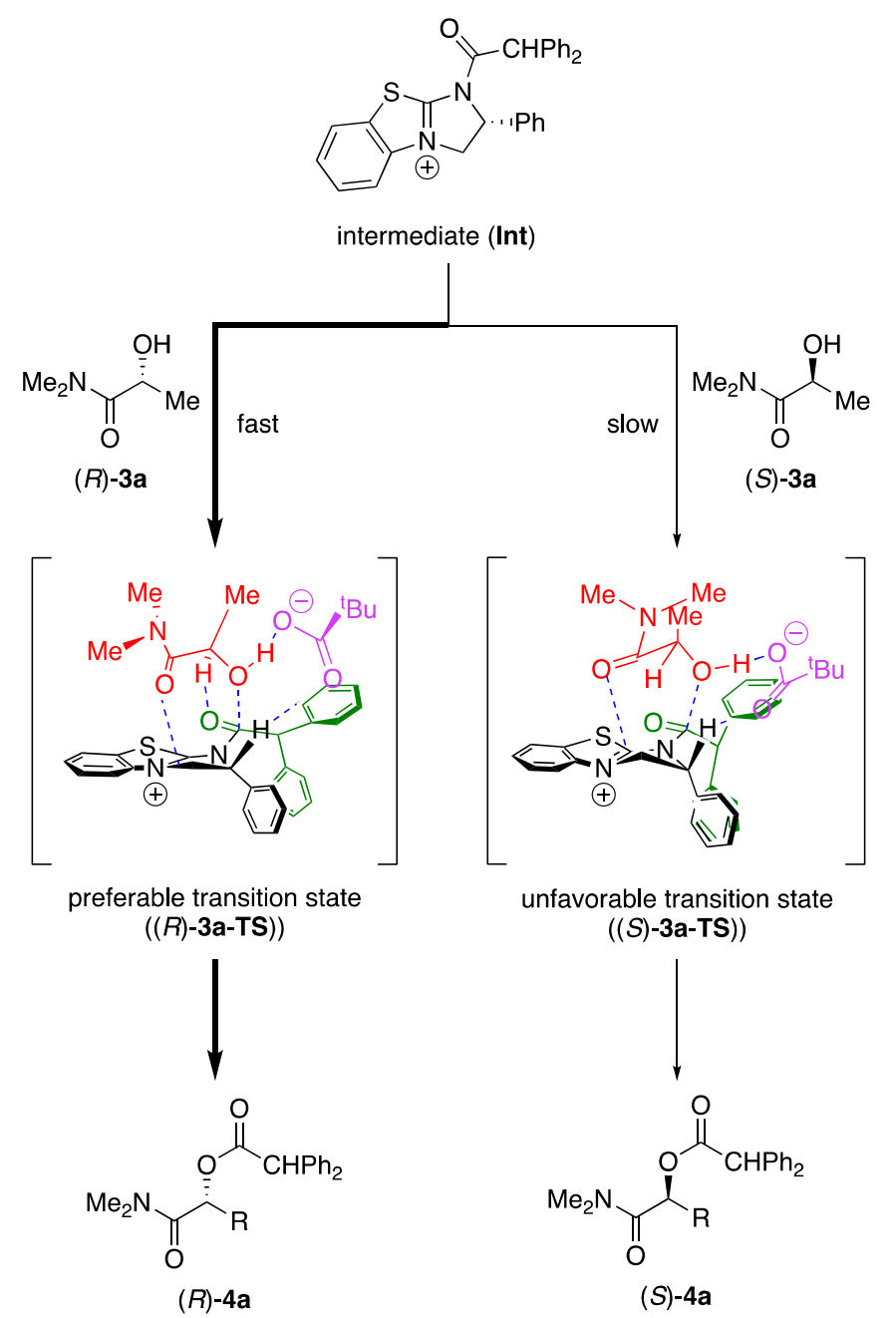

Scheme 2. Calculated transition states with a kinetic resolution (KR) of $( \pm)-3$.

The most stable transition state that affords $(R)$ - or (S)-2-acyloxy-dimethylamides is shown in Figure 1. It was found that the high selectivity attained in the present KR can be explained by the rapid transformation of $(R)-3$ into $(R)-4$ through the stabilized transition state $(R)-3-T S$, which consists of $(R)-3$ and the isothiouronium salt derived from the mixed anhydride and $(R)-\mathrm{BTM}$. The formation of a $\mathrm{C}-\mathrm{O}$ bond (between carbonyl carbon of the acid component and oxygen of the hydroxy group) at a distance of $2.086 \AA$ is accompanied by the coordination of oxygen in the carbonyl moiety to hydrogen at the C-2 position of the 2-hydroxydimethylamide at a distance of $2.342 \AA$, as shown in Figure 1 . It was further observed that the length of the cleaved $\mathrm{O}-\mathrm{H}$ bond (between oxygen and hydrogen in the hydroxyl group) was $1.356 \AA$. A frequency analysis of $(R)-3$-TS revealed that the nucleophilic attack of the alcohol to the carbonyl group and the deprotonation of the hydroxyl group with the pivalate anion proceeded via a concerted reaction mechanism because the $\mathrm{C}-\mathrm{O}$ bond-forming step and the $\mathrm{O}-\mathrm{H}$ bond-cleaving process occurred simultaneously.

An attractive interaction occurred between oxygen in the amide carbonyl group and the positive electronic charge on the surface of the thiouronium salt, together with coordination of oxygen in the pivalate anion to hydrogen in the hydroxyl group (1.109 $\AA$ ) and hydrogen at the 
C-2 position of the dihydroimidazolium salt (2.964 $\AA$ ). However, complexation of the thiouronium salt with $(R)$-2-hydroxydimethylamide ((R)-3a), an enantiomer of (S)-2-hydroxydimethylamide ((S)-3a), produced an unstable structure, i.e., (S)-3a-TS; thus, the formation of (S)-3a-TS proceeded slowly due to an energy gap of $4.02 \mathrm{kcal} / \mathrm{mol}$.

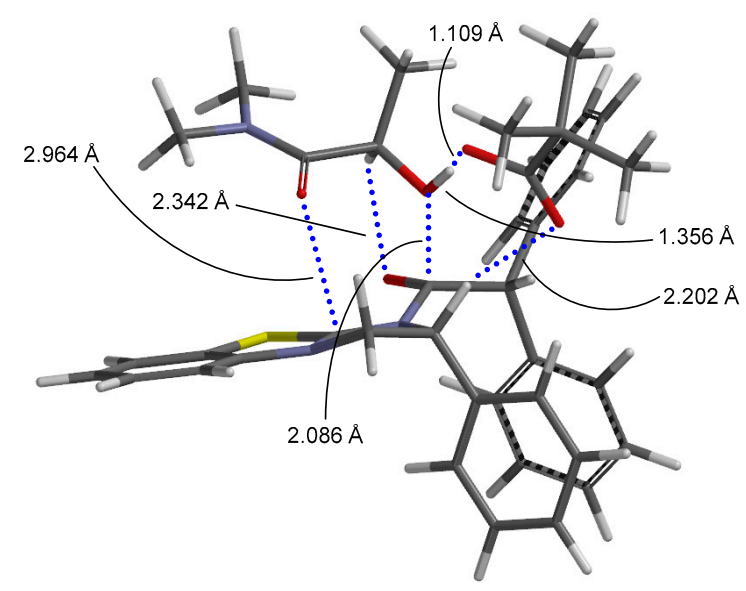

Preferable transition structure ((R)-3a-TS)

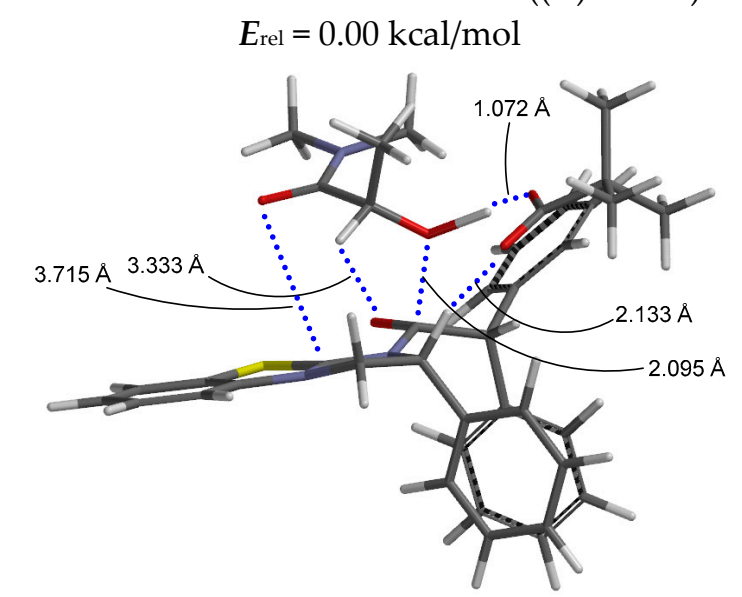

Unfavorable transition state structure ((S)-3a-TS)

$$
E_{\text {rel }}=4.02 \mathrm{kcal} / \mathrm{mol}
$$

Figure 1. Three-dimensional structures of the calculated transition states ((R)-3a-TS and (S)-3a-TS).

We performed further calculations on the KR of 2-hydroxy-Weinreb amides (Scheme 3). The most stable transition state that affords $(R)$ - or $(S)$-2-acyloxy-Weinreb amides is shown in Figure 2 [34]. It was found that the high selectivity attained in the present KR can be explained by the rapid transformation of $(R)-5$ to $(R)-6$ through the stabilized transition state $(R)-5-\mathrm{TS}$, which consists of $(R)-5$ and the isothiouronium salt derived from the mixed anhydride and (R)-BTM. The formation of a C-O bond (between carbonyl carbon of the acid component and oxygen of the hydroxy group) at a distance of $2.080 \AA$ is accompanied by the coordination of oxygen in the carbonyl moiety to hydrogen at the C-2 position of the 2-hydroxy-Weinreb amide at a distance of $2.311 \AA$, as shown in Figure 2. It was further observed that the length of the cleaved $\mathrm{O}-\mathrm{H}$ bond (between oxygen and hydrogen in the hydroxy group) was $1.396 \AA$. A frequency analysis of $(R)$-5-TS revealed that the nucleophilic attack of the alcohol to the carbonyl group and the deprotonation of the hydroxyl group with the pivalate anion proceeded via a concerted reaction mechanism as for the reaction with the 2-hydroxy dimethylamide. 


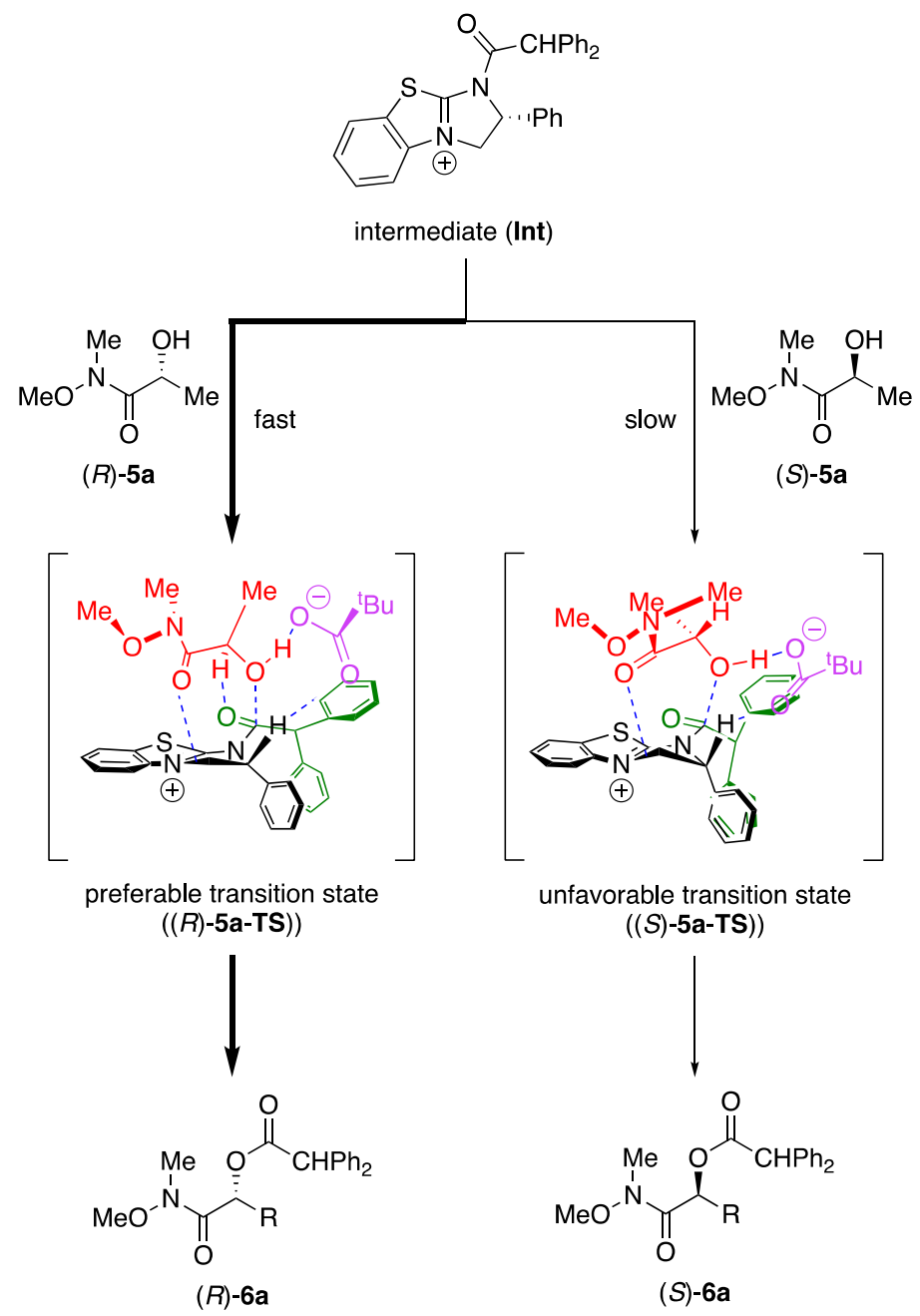

Scheme 3. Calculated transition states with a KR of $( \pm)-5$.

An attractive interaction occurred between oxygen in the amide carbonyl group and the positive electronic charge on the surface of the thiouronium salt, together with coordination of oxygen in the pivalate anion to hydrogen in the hydroxyl group $(1.088 \AA)$ and hydrogen at the C-2 position of the dihydroimidazolium salt (2.928 $\AA$ ). However, complexation of the thiouronium salt with $(R)$-2-hydroxy-Weinreb amide $((R)-5 \mathbf{a})$, an enantiomer of (S)-2-hydroxy-Weinreb amide [(S)-5a], produced an unstable structure, i.e., (S)-5a-TS; thus, the formation of (S)-5a-TS proceeded slowly due to an energy gap of $3.24 \mathrm{kcal} / \mathrm{mol}$.

Finally, we attempted to transform the obtained chiral 2-hydroxyamides and their esters based on the KR to demonstrate the synthetic utility of this method (Scheme 4). Reduction of chiral 2-hydroxy- $\mathrm{N}, \mathrm{N}$-dimethylamide (S)-1d with $\mathrm{BH}_{3} \cdot \mathrm{SMe}_{2}$ in THF afforded amino alcohol (S)-7 in good yield with no loss of chirality (i). When 2-acyloxy-N,N-dimethylamide (R)-2d was subjected to solvolysis with $\mathrm{K}_{2} \mathrm{CO}_{3}$ in $\mathrm{MeOH}$, 2-hydroxyamide $(R)$-1d was produced in good yield (ii). Treatment of 2-hydroxy-Weinreb amide (S)-5a and 2-ayloxy-Weinreb amide (R)-6a with PhMgBr afforded the corresponding 2-hydroxyketone 8 with opposite stereochemistry (iii) and (iv), respectively. 


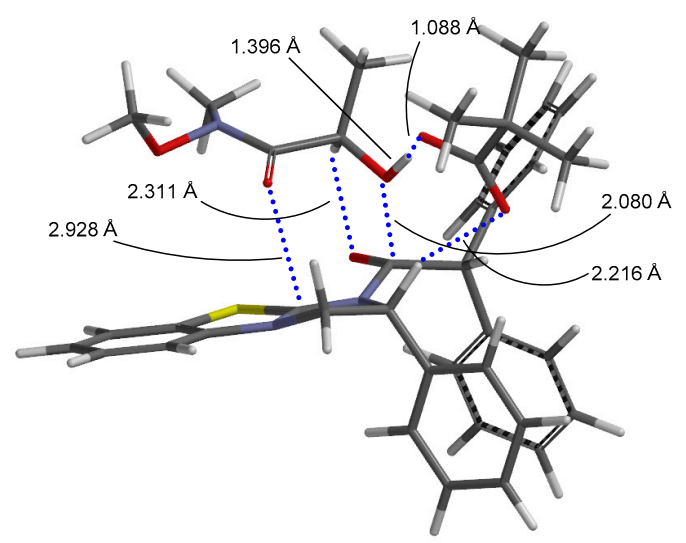

Preferable transition structure $((R)-5 \mathbf{a}-\mathrm{TS})$ $E_{\text {rel }}=0.00 \mathrm{kcal} / \mathrm{mol}$

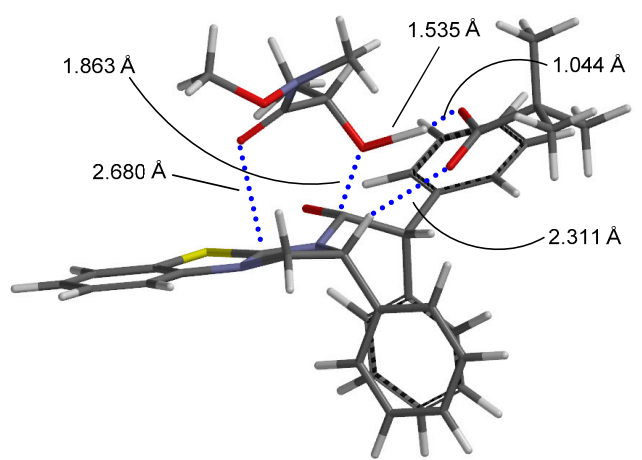

Unfavorable transition structure $[(S)-5 a-T S]$ $E_{\text {rel }}=3.24 \mathrm{kcal} / \mathrm{mol}$

Figure 2. Three-dimensional structures of the calculated transition states $((R)-\mathbf{5 a}-\mathbf{T S}$ and $(S)-\mathbf{5 a}-\mathbf{T S})$.<smiles>CNC(=O)C(O)CCc1ccccc1</smiles>

(S)-1d; >99\% ee

$$
\mathrm{MeO}^{-\mathrm{N}} \prod_{\mathrm{O}}^{\mathrm{Me}} \frac{\operatorname{PhMgBr}(3.0 \text { eq. })}{\operatorname{THF}(0.1 \mathrm{M}), 0^{\circ} \mathrm{C}, 3 \mathrm{~h}}
$$

(S)-5a; $98 \%$ ee<smiles>CC(OC(=O)C=Cc1ccccc1)C(=O)N(C)C</smiles><smiles>CN(C)CC(O)Cc1ccccc1</smiles>

(S)-7; 71\%, >99\% ee<smiles>CN(C)C(=O)C(O)Cc1ccccc1</smiles>

(S)-8; quant., $84 \%$ ee<smiles>CC(O)C(=O)c1ccccc1</smiles>

(S)-8; quant., $99 \%$ ee<smiles>CC(O)C(=O)c1ccccc1</smiles>

(R)-8; $98 \%, 93 \%$ ee

Scheme 4. Transformation of chiral 2-hydroxyamides and their esters. 


\section{Materials and Methods}

\subsection{General Information}

Optical rotations were determined using a Jasco P-1020 polarimeter. Infrared (IR) spectra were obtained using a Jasco FT/IR-4600 Fourier transform infrared spectrometer. Proton and carbon nuclear magnetic resonance $\left({ }^{1} \mathrm{H}\right.$ and $\left.{ }^{13} \mathrm{C} \mathrm{NMR}\right)$ spectra were recorded with chloroform $\left(\mathrm{in}_{\mathrm{CDCl}}\right)$ on the following instruments: JEOL JNM-AL500 $\left({ }^{1} \mathrm{H}\right.$ at $500 \mathrm{MHz}$ and ${ }^{13} \mathrm{C}$ at $\left.125 \mathrm{MHz}\right)$. Mass spectra were determined by a Bruker Daltonics micrOTOF focus (ESI-TOF) mass spectrometer. Thin layer chromatography was performed on Wakogel B5F. HPLC was performed with a Hitachi LaChrom Elite system composed of the Organizer, L-2400 UV Detector, and L-2130 Pump.

\subsection{Typical Procedure for the Preparation of Optically Active 2-Hydroxy-dimethylamides $\mathbf{2 d}$ and $\mathbf{4 a - 4 k}$}

Condition A; Asymmetric esterification of racemic 2-hydroxy-dimethylamide $(( \pm)$-1d) with diphenylacetic acid by using $\mathrm{Piv}_{2} \mathrm{O}$ in the presence of $(R)$-BTM was described (Table 1, entry 4): To a solution of racemic 2-hyroxy-dimethylamide $(( \pm)-1 d)(41.5 \mathrm{mg}, 0.20 \mathrm{mmol})$ in $\mathrm{Et}_{2} \mathrm{O}(1.0 \mathrm{~mL}$, $0.20 \mathrm{M})$ at room temperature were successively added diphenylacetic acid $(31.8 \mathrm{mg}, 0.15 \mathrm{mmol}), \mathrm{Piv} 2 \mathrm{O}$ $(36.5 \mu \mathrm{L}, 0.18 \mathrm{mmol}), i-\operatorname{Pr}_{2} \mathrm{NEt}(62.7 \mu \mathrm{L}, 0.36 \mathrm{mmol})$ and $(R)-\mathrm{BTM}(2.5 \mathrm{mg}, 0.01 \mathrm{mmol})$. The reaction mixture was stirred for $24 \mathrm{~h}$ at the same temperature and then it was quenched with saturated aqueous $\mathrm{NaHCO}_{3}$. The organic layer was separated, and the aqueous layer was extracted with EtOAc. The combined organic layer was dried over $\mathrm{Na}_{2} \mathrm{SO}_{4}$. After filtration of the mixture and evaporation of the solvent, the crude product was purified by preparative thin layer chromatography on silica to afford the corresponding optically active ester $(R)-2 \mathrm{~d}(38.2 \mathrm{mg}, 48 \%$ yield, $92 \%$ ee) and the recovered optically active alcohol $(S)-\mathbf{1 d}(19.1 \mathrm{mg}, 46 \%$ yield, 99\% ee) [ $s=254$, Table 1, Entry 4].

Condition B; Asymmetric esterification of racemic 2-hydroxy-dimethylamide $(( \pm)$-1d) with diphenylacetic anhydride in the presence of (R)-BTM was described (Table 1, entry 6): To a solution of racemic 2-hydroxy-dimethylamide $(( \pm)-1 d)(41.5 \mathrm{mg}, 0.20 \mathrm{mmol})$ in $\mathrm{Et}_{2} \mathrm{O}(1.0 \mathrm{~mL}, 0.2 \mathrm{M})$ at room temperature were successively added diphenylacetic anhydride (48.8 mg, $0.12 \mathrm{mmol}), i-\operatorname{Pr}_{2} \mathrm{NEt}(20.9 \mu \mathrm{L}$, $0.12 \mathrm{mmol})$ and $(R)-B T M(2.5 \mathrm{mg}, 0.011 \mathrm{mmol})$. The reaction mixture was stirred for $24 \mathrm{~h}$ at the same temperature and then it was quenched with saturated aqueous $\mathrm{NaHCO}_{3}$. The organic layer was separated, and the aqueous layer was extracted with EtOAc. The combined organic layer was dried over $\mathrm{Na}_{2} \mathrm{SO}_{4}$. After filtration of the mixture and evaporation of the solvent, the crude product was purified by preparative thin layer chromatography on silica to afford the corresponding optically active ester $(R)-2 d(37.4 \mathrm{mg}, 47 \%$ yield, 91\% ee) and the recovered optically active alcohol (S)-1d (20.7 $\mathrm{mg}, 50 \%$ yield, $98 \%$ ee) [ $s=95$, Table 1, Entry 6].

(S)-2-Hydroxy-N-methyl-4-phenylbutanamide ((S)-1a). HPLC (CHIRALPAK IC, $i$-PrOH/hexane = 1/20, flow rate $=1.0 \mathrm{~mL} / \mathrm{min}): t \mathrm{R}=47.2 \mathrm{~min}(57.2 \%), t \mathrm{R}=54.5 \mathrm{~min}(42.8 \%)$; IR (neat): 3309, 1643, 1619, $1550 \mathrm{~cm}^{-1} ;{ }^{1} \mathrm{H}$ NMR $\left(\mathrm{CDCl}_{3}\right): \delta 7.33-7.22$ (m, 5H, Ph), 6.85 (brs, 1H, NH), 4.15 (m, 1H, 2-H), 3.92 $(\mathrm{d}, J=5.5 \mathrm{~Hz}, 1 \mathrm{H}, \mathrm{OH}), 2.86$ (s, 3H, NMe), 2.86-2.79 (m, 2H, 4-H), 2.24-2.12 (m, 1H, 3-H), $2.02-1.95$ $(\mathrm{m}, 1 \mathrm{H}, 3-\mathrm{H}) ;{ }^{13} \mathrm{C}$ NMR $\left(\mathrm{CDCl}_{3}\right): \delta 174.8,141.2,128.4,126.0,71.4,36.3,31.2,25.7$; HR MS: calcd for $\mathrm{C}_{12} \mathrm{H}_{17} \mathrm{NO}_{2} \mathrm{Na}[\mathrm{M}+\mathrm{Na}]^{+} 216.0995$, found 216.1004 .

(S)-N-Benzyl-2-hydroxy-4-phenylbutanamide ((S)-1b). HPLC (CHIRALPAK IC, $i$-PrOH/hexane = 1/9, flow rate $=1.0 \mathrm{~mL} / \mathrm{min}): t \mathrm{R}=12.3 \mathrm{~min}(42.8 \%), t \mathrm{R}=14.6 \mathrm{~min}(57.2 \%)$; $\mathrm{IR}(\mathrm{KBr}): 3366,3252,1621,1538$, 1496, 1454, 732, $699 \mathrm{~cm}^{-1} ;{ }^{1} \mathrm{H}$ NMR $\left(\mathrm{CDCl}_{3}\right): \delta 7.37-7.18(\mathrm{~m}, 10 \mathrm{H}, \mathrm{Ph}), 7.02$ (brs, 1H, NH), 4.46 (dd, $J=15.0,6.0 \mathrm{~Hz}, 1 \mathrm{H}, \mathrm{Bn}), 4.42(\mathrm{dd}, J=15.0,6.0 \mathrm{~Hz}, 1 \mathrm{H}, \mathrm{Bn}), 4.16(\mathrm{ddd}, J=8.0,5.0,3.5 \mathrm{~Hz}, 1 \mathrm{H}, 2-\mathrm{H})$, 3.47 (brs, 1H, OH), 2.83-2.73 (m, 2H, 4-H), 2.25-2.15 (m, 1H, 3-H), 2.04-1.94 (m, 1H, 3-H); ${ }^{13} \mathrm{C} \mathrm{NMR}$ $\left(\mathrm{CDCl}_{3}\right): \delta 173.8,141.1,137.8,128.7,128.4,127.6,127.5,126.0,71.5,43.1,36.4,31.2 ; \mathrm{HR} \mathrm{MS}$ : calcd for $\mathrm{C}_{17} \mathrm{H}_{19} \mathrm{NO}_{2} \mathrm{Na}[\mathrm{M}+\mathrm{Na}]^{+} 292.1308$, found 292.1312 . 
(S)-2-Hydroxy-N,4-diphenylbutanamide ((S)-1c). HPLC (CHIRALPAK IC, $i$-PrOH $/$ hexane = 1/9, flow rate $=1.0 \mathrm{~mL} / \mathrm{min}): t \mathrm{R}=8.2 \mathrm{~min}(86.6 \%), t \mathrm{R}=11.4 \mathrm{~min}(13.4 \%) ; \mathrm{IR}(\mathrm{KBr}): 3332,3230,1656,1496,1445$, 755, $702 \mathrm{~cm}^{-1},{ }^{1} \mathrm{H}$ NMR $\left(\mathrm{CDCl}_{3}\right): \delta 8.42$ (s, 1H, NH), 7.57-7.48 (m, 2H, Ph), 7.31-7.09 (m, 8H, Ph), 4.24 $(\mathrm{ddd}, J=8.3,4.8,4.0 \mathrm{~Hz}, 1 \mathrm{H}, 2-\mathrm{H}), 2.89(\mathrm{brd}, J=4.0 \mathrm{~Hz}, 1 \mathrm{H}, \mathrm{OH}), 2.82(\mathrm{~d}, J=8.0 \mathrm{~Hz}, 1 \mathrm{H}, 4-\mathrm{H}), 2.81(\mathrm{~d}$, $J=9.5 \mathrm{~Hz}, 1 \mathrm{H}, 4-\mathrm{H}) 2.32-2.22(\mathrm{~m}, 1 \mathrm{H}, 3-\mathrm{H}), 2.11-2.01(\mathrm{~m}, 1 \mathrm{H}, 3-\mathrm{H}) ;{ }^{13} \mathrm{C} \mathrm{NMR}\left(\mathrm{CDCl}_{3}\right): \delta 171.6,140.9$, 137.1, 129.1, 128.6, 128.5, 126.2, 124.6, 119.8, 72.1, 36.2, 31.3; HR MS: calcd for $\mathrm{C}_{16} \mathrm{H}_{17} \mathrm{NO}_{2} \mathrm{Na}[\mathrm{M}+\mathrm{Na}]^{+}$ 278.1151 , found 278.1153 .

(S)-2-Hydroxy-N,N-dimethyl-4-phenylbutanamide ((S)-1d). HPLC (CHIRALPAK IC, $i$-PrOH $/$ hexane = $1 / 4$, flow rate $=1.0 \mathrm{~mL} / \mathrm{min}): t \mathrm{R}=29.2 \mathrm{~min}(100.0 \%) ; \mathrm{IR}$ (neat): 3457, 1738, 1498, 1456, 1045, 752, 698 $\mathrm{cm}^{-1} ;{ }^{1} \mathrm{H}$ NMR $\left(\mathrm{CDCl}_{3}\right): \delta 7.31-7.16(\mathrm{~m}, 5 \mathrm{H}, \mathrm{Ph}), 4.30(\mathrm{ddd}, J=9.0,7.5,3.0 \mathrm{~Hz}, 1 \mathrm{H}, 2-\mathrm{H}), 3.78(\mathrm{dd}$, $J=7.5,1.5 \mathrm{~Hz}, 1 \mathrm{H}, \mathrm{OH}), 2.96$ (s, 3H, OMe), 2.87-2.75 (m, 2H, 4-H), 2.80 (s, 3H, NMe), 1.91 (dddd, $J=13.5,9.0,8.0,3.0 \mathrm{~Hz}, 1 \mathrm{H}, 3-\mathrm{H}), 1.78(\mathrm{dddd}, J=13.5,9.0,8.5,5.0 \mathrm{~Hz}, 1 \mathrm{H}, 3-\mathrm{H}) ;{ }^{13} \mathrm{C} \mathrm{NMR}\left(\mathrm{CDCl}_{3}\right): \delta$ 174.2, 141.3, 128.6, 128.4, 126.0, 66.9, 36.4, 36.1, 35.8, 31.2; HR MS: calcd for $\mathrm{C}_{12} \mathrm{H}_{17} \mathrm{NO}_{2} \mathrm{Na}[\mathrm{M}+\mathrm{Na}]^{+}$ 230.1151 , found 230.1150 .

(S)-2-Hydroxy-N-methoxy-N-methyl-4-phenylbutanamide ((S)-1e) (=(S)-5h). HPLC (CHIRALPAK IC, $i-\mathrm{PrOH} /$ hexane $=1 / 9$, flow rate $=1.0 \mathrm{~mL} / \mathrm{min}): t \mathrm{R}=26.7 \mathrm{~min}(0.8 \%), t \mathrm{R}=29.6 \mathrm{~min}(99.2 \%)$; IR (neat): 3439, 1657, 1487, 1450, 753, $707 \mathrm{~cm}^{-1} ;{ }^{1} \mathrm{H}$ NMR $\left(\mathrm{CDCl}_{3}\right): \delta 7.36-7.19(\mathrm{~m}, 5 \mathrm{H}, \mathrm{Ph}), 4.38(\mathrm{dd}, J=7.0,7.0$ $\mathrm{Hz}, 1 \mathrm{H}, 2-\mathrm{H}), 3.59$ (s, 3H, OMe), 3.40 (d, J = 7.0 Hz, 1H, OH), 3.24 (s, 3H, NMe), 2.88 (ddd, J = 14.0, 9.0, $5.0 \mathrm{~Hz}, 1 \mathrm{H}, 4-\mathrm{H}), 2.83$ (ddd, $J=14.0,8.5,8.5 \mathrm{~Hz}, 1 \mathrm{H}, 4-\mathrm{H}), 2.16-2.05$ (m, 1H, 3-H), 1.90-1.83 (m, 1H, 3-H); ${ }^{13} \mathrm{C} \mathrm{NMR}\left(\mathrm{CDCl}_{3}\right): \delta 175.0,141.4,128.6,128.3,125.8,67.7,61.1,36.1,32.4,31.2 ; \mathrm{HR}$ MS: calcd for $\mathrm{C}_{12} \mathrm{H}_{17} \mathrm{NO}_{3} \mathrm{Na}[\mathrm{M}+\mathrm{Na}]^{+} 246.1101$, found 246.1106 .

(R)-2-(Diphenylacetyloxy)-N-methyl-4-phenylbutanamide ((R)-2a). HPLC (CHIRALPAK IC, $i$-PrOH/ hexane $=2 / 3$, flow rate $=0.5 \mathrm{~mL} / \mathrm{min}): t \mathrm{R}=23.6 \mathrm{~min}(55.9 \%), t \mathrm{R}=39.3 \mathrm{~min}(44.1 \%)$; IR (neat): 3424, 3309, 1743, 1673, 1542, 748, $709 \mathrm{~cm}^{-1} ;{ }^{1} \mathrm{H}$ NMR $\left(\mathrm{CDCl}_{3}\right): \delta 7.32-7.20$ (m, 10H, Ph), 7.17-7.07 (m, 3H, Ph), 6.99-6.97 (m, 2H, Ph), 5.41 (brs, 1H, NH), 5.23 (dd, J = 7.0, 4.0 Hz, 1H, 2-H), 4.99 (s, 1H, $\left.\mathrm{CHPh}_{2}\right), 2.52-2.45$ (m, 2H, 3-H), 2.49 (s, 3H, NMe), 2.18-2.00 (m, 1H, 4-H); ${ }^{13} \mathrm{C} \mathrm{NMR}\left(\mathrm{CDCl}_{3}\right): \delta 170.7$, 169.8, 140.6, 137.8, 137.6, 128.9, 128.7, 128.6, 128.6, 128.4, 128.3, 127.7, 127.6, 126.0, 73.9, 57.1, 33.3, 31.0, 25.7; HR MS: calcd for $\mathrm{C}_{26} \mathrm{H}_{27} \mathrm{NO}_{3} \mathrm{Na}[\mathrm{M}+\mathrm{Na}]^{+} 410.1727$, found 410.1717 .

(R)-N-Benzyl-2-(diphenylacetyloxy)-4-phenylbutanamide ((R)-2b). HPLC (CHIRALPAK AD-H, $i$-PrOH/ hexane $=1 / 9$, flow rate $=1.0 \mathrm{~mL} / \mathrm{min}): t \mathrm{R}=23.1 \mathrm{~min}(58.6 \%), t \mathrm{R}=25.7 \mathrm{~min}(41.4 \%)$; IR (neat): 3308 , 1744, 1677, 1496, 1451, 747, $697 \mathrm{~cm}^{-1} ;{ }^{1} \mathrm{H}$ NMR $\left(\mathrm{CDCl}_{3}\right): \delta 7.36-7.18(\mathrm{~m}, 16 \mathrm{H}, \mathrm{Ph}), 7.14-7.07(\mathrm{~m}, 4 \mathrm{H}$, $\mathrm{Ph}), 5.88(\mathrm{t}, J=5.5 \mathrm{~Hz}, 1 \mathrm{H}, \mathrm{NH}), 5.40(\mathrm{dd}, J=7.3,4.3 \mathrm{~Hz}, 1 \mathrm{H}, 2-\mathrm{H}), 5.05\left(\mathrm{~s}, 1 \mathrm{H}, 2^{\prime}-\mathrm{H}\right), 4.32(\mathrm{dd}, J=14.8$, $5.5 \mathrm{~Hz}, 1 \mathrm{H}, \mathrm{Bn}), 4.24(\mathrm{dd}, J=14.8,5.5 \mathrm{~Hz}, 1 \mathrm{H}, \mathrm{Bn}), 2.62(\mathrm{t}, J=8.3 \mathrm{~Hz}, 2 \mathrm{H}, 4-\mathrm{H}), 2.32-2.17(\mathrm{~m}, 2 \mathrm{H}, 3-\mathrm{H})$; ${ }^{13} \mathrm{C} \mathrm{NMR}\left(\mathrm{CDCl}_{3}\right): \delta 170.7,169.2,140.6,137.7,137.6,137.6,128.9,128.7,128.6,128.6,128.4,128.4,128.3$, $127.7,127.6,127.6,127.5,126.1,73.9,57.1,43.0,33.4,31.0 ; \mathrm{HR}$ MS: calcd for $\mathrm{C}_{31} \mathrm{H}_{29} \mathrm{NO}_{3} \mathrm{Na}[\mathrm{M}+\mathrm{Na}]^{+}$ 486.2040 , found 486.2031 .

(R)-2-(Diphenylacetyloxy)-N,4-diphenylbutanamide ((R)-2c). HPLC (CHIRALPAK IC, $i$-PrOH $/$ hexane = $1 / 9$, flow rate $=1.0 \mathrm{~mL} / \mathrm{min}): t \mathrm{R}=11.5 \mathrm{~min}(10.7 \%), t \mathrm{R}=25.6 \mathrm{~min}(89.3 \%) ; \mathrm{IR}$ (neat): 3312, 1750, 1670, 1494, 1447, 754, $695 \mathrm{~cm}^{-1} ;{ }^{1} \mathrm{H}$ NMR $\left(\mathrm{CDCl}_{3}\right): \delta 7.34-7.00(\mathrm{~m}, 21 \mathrm{H}, \mathrm{Ph}, \mathrm{NH}), 5.40(\mathrm{dd}, J=6.8,4.5 \mathrm{~Hz}, 1 \mathrm{H}$, 2-H), 5.03 (s, 1H, 2'-H), 2.60 (t, $J=8.0 \mathrm{~Hz}, 2 \mathrm{H}, 4-\mathrm{H}), 2.23(\mathrm{~m}, 2 \mathrm{H}, 3-\mathrm{H}) ;{ }^{13} \mathrm{C} \mathrm{NMR}\left(\mathrm{CDCl}_{3}\right): \delta 170.6,167.3$, 140.5, 137.7, 137.7, 136.6, 129.2, 128.8, 128.8, 128.7, 128.5, 128.5, 128.3, 127.8, 127.7, 126.1, 124.7, 119.9, 73.9, 57.2, 33.3, 31.0; HR MS: calcd for $\mathrm{C}_{30} \mathrm{H}_{27} \mathrm{NO}_{3} \mathrm{Na}[\mathrm{M}+\mathrm{Na}]^{+} 472.1883$, found 472.1874 .

(R)-2-(Diphenylacetyloxy)-N,N-dimethyl-4-phenylbutanamide ((R)-2d). HPLC (CHIRALPAK IC, $i$-PrOH/ hexane $=1 / 9$, flow rate $=1.0 \mathrm{~mL} / \mathrm{min}): t \mathrm{R}=17.9 \mathrm{~min}(4.3 \%), t \mathrm{R}=40.2 \mathrm{~min}(95.7 \%) ; \mathrm{IR}$ (neat): 1737, 1663, 1496, 744, $697 \mathrm{~cm}^{-1} ;{ }^{1} \mathrm{H}$ NMR $\left(\mathrm{CDCl}_{3}\right): \delta 7.38-7.07(\mathrm{~m}, 13 \mathrm{H}, \mathrm{Ph}), 6.92-6.85(\mathrm{~m}, 2 \mathrm{H}, \mathrm{Ph}), 5.13(\mathrm{~s}$, 
$\left.1 \mathrm{H}, 2^{\prime}-\mathrm{H}\right), 5.08(\mathrm{dd}, J=10.0,3.5 \mathrm{~Hz}, 1 \mathrm{H}, 2-\mathrm{H}), 2.84(\mathrm{~s}, 3 \mathrm{H}, \mathrm{OMe}), 2.73$ (s, 3H, NMe), 2.59 (ddd, $J=14.0$, 8.5, $5.0 \mathrm{~Hz}, 1 \mathrm{H}, 4-\mathrm{H}), 2.41$ (ddd, $J=14.0,8.5,8.5 \mathrm{~Hz}, 1 \mathrm{H}, 4-\mathrm{H}), 2.11$ (dddd, $J=14.5,10.0,8.5,5.0 \mathrm{~Hz}$, $1 \mathrm{H}, 3-\mathrm{H}), 1.88$ (dddd, $J=14.5,8.5,8.5,3.5 \mathrm{~Hz}, 1 \mathrm{H}, 3-\mathrm{H}) ;{ }^{13} \mathrm{C} \mathrm{NMR}\left(\mathrm{CDCl}_{3}\right): \delta 172.3,169.4,140.3,138.5$, 138.4, 128.8, 128.7, 128.5, 128.4, 128.3, 127.4, 127.2, 126.2, 70.1, 56.7, 36.5, 35.9, 32.4, 31.0; HR MS: calcd for $\mathrm{C}_{26} \mathrm{H}_{27} \mathrm{NO}_{3} \mathrm{Na}[\mathrm{M}+\mathrm{Na}]^{+} 424.1883$, found 424.1901.

(R)-2-(Diphenylacetyloxy)-N-methoxy-N-methyl-4-phenylbutanamide ((R)-2e) (=(R)-6h). HPLC (CHIRALPAK $\mathrm{IC}, i-\mathrm{PrOH} /$ hexane $=1 / 9$, flow rate $=1.0 \mathrm{~mL} / \mathrm{min}): t \mathrm{R}=14.8 \mathrm{~min}(3.0 \%), t \mathrm{R}=41.3 \mathrm{~min}(97.0 \%) ; \mathrm{IR}$ (neat): 1736, 1674, 1496, 1450, 741, $702 \mathrm{~cm}^{-1},{ }^{1} \mathrm{H}$ NMR $\left(\mathrm{CDCl}_{3}\right): \delta 7.51-7.19(\mathrm{~m}, J=13 \mathrm{H}, \mathrm{Ph}), 7.05-6.99$ (m, 2H, Ph), $5.27\left(\mathrm{~s}, 1 \mathrm{H}, 2^{\prime}-\mathrm{H}\right), 5.19(\mathrm{dd}, J=9.5,3.5 \mathrm{~Hz}, 1 \mathrm{H}, 2-\mathrm{H}), 3.61(\mathrm{~s}, 3 \mathrm{H}, \mathrm{OMe}), 3.21(\mathrm{~s}, 3 \mathrm{H}, \mathrm{NMe})$, $2.74(\mathrm{ddd}, J=14.0,8.0,5.0 \mathrm{~Hz}, 1 \mathrm{H}, 4-\mathrm{H}), 2.53(\mathrm{ddd}, J=14.0,8.5,8.5 \mathrm{~Hz}, 1 \mathrm{H}, 4-\mathrm{H}), 2.22-2.07(\mathrm{~m}, 2 \mathrm{H}$, $3-\mathrm{H}) ;{ }^{13} \mathrm{C} \mathrm{NMR}\left(\mathrm{CDCl}_{3}\right): \delta 172.5,170.0,140.3,138.5,138.4,128.8,128.8,128.6,128.4,128.4,128.3,127.3$, 127.1, 126.0, 70.8, 61.1, 56.8, 32.1, 31.7, 31.1; HR MS: calcd for $\mathrm{C}_{26} \mathrm{H}_{27} \mathrm{NO}_{4} \mathrm{Na}[\mathrm{M}+\mathrm{Na}]^{+} 440.1832$, found 440.1852 .

(S)-2-Hydroxy-N,N-dimethylpropanamide ((S)-3a). HPLC (CHIRALPAK IC, $i$-PrOH $/$ hexane $=1 / 4$, flow rate $=1.0 \mathrm{~mL} / \mathrm{min}): t \mathrm{R}=17.3 \mathrm{~min}(5.7 \%), t \mathrm{R}=26.6 \mathrm{~min}(93.3 \%) ; \mathrm{IR}$ (neat): $3417,1643 \mathrm{~cm}^{-1} ;{ }^{1} \mathrm{H} \mathrm{NMR}$ $\left(500 \mathrm{MHz}, \mathrm{CDCl}_{3}\right): \delta 4.41(\mathrm{q}, J=6.5 \mathrm{~Hz}, 1 \mathrm{H}, 2-\mathrm{H}), 3.82(\mathrm{br} \mathrm{s}, 1 \mathrm{H}, \mathrm{OH}) 2.95(\mathrm{~s}, 3 \mathrm{H}, \mathrm{NMe}), 2.94(\mathrm{~s}, 3 \mathrm{H}$, $\mathrm{NMe}), 1.27(\mathrm{~d}, J=6.5 \mathrm{~Hz}, 3 \mathrm{H}, 3-\mathrm{H}) ;{ }^{13} \mathrm{C} \mathrm{NMR}\left(125 \mathrm{MHz}, \mathrm{CDCl}_{3}\right): \delta 174.9,64.0,36.2,35.8,20.8$; HR MS: calcd for $\mathrm{C}_{5} \mathrm{H}_{11} \mathrm{NO}_{2} \mathrm{Na}[\mathrm{M}+\mathrm{Na}]^{+} 140.0682$, found 140.0684 .

(S)-2-Hydroxy-N,N-dimethylbutanamide ((S)-3b). HPLC (CHIRALPAK IC, $i$-PrOH $/$ hexane $=1 / 4$, flow rate $=1.0 \mathrm{~mL} / \mathrm{min}): t \mathrm{R}=15.6 \mathrm{~min}(3.7 \%), t \mathrm{R}=28.2 \mathrm{~min}(96.3 \%) ; \mathrm{IR}$ (neat): $3425,1642 \mathrm{~cm}^{-1} ;{ }^{1} \mathrm{H} \mathrm{NMR}$ $\left(500 \mathrm{MHz}, \mathrm{CDCl}_{3}\right): \delta 4.27(\mathrm{~m}, 1 \mathrm{H}, 2-\mathrm{H}), 3.68(\mathrm{~d}, J=7.5 \mathrm{~Hz}, 1 \mathrm{H}, \mathrm{OH}), 2.96(\mathrm{~s}, 3 \mathrm{H}, \mathrm{NMe}), 2.94(\mathrm{~s}, 3 \mathrm{H}$, $\mathrm{NMe}), 1.67(\mathrm{~m}, 1 \mathrm{H}, 3-\mathrm{H}), 1.46(\mathrm{~m}, 1 \mathrm{H}, 3-\mathrm{H}), 0.94(\mathrm{dd}, J=7.0,7.0 \mathrm{~Hz}, 3 \mathrm{H}, 4-\mathrm{H}) ;{ }^{13} \mathrm{C} \mathrm{NMR}(125 \mathrm{MHz}$, $\left.\mathrm{CDCl}_{3}\right): \delta 174.2,68.9,36,3,35,7,27,5,9.1 ;$ HR MS: calcd for $\mathrm{C}_{6} \mathrm{H}_{13} \mathrm{NO}_{2} \mathrm{Na}[\mathrm{M}+\mathrm{Na}]^{+} 154.0838$, found 154.0845 .

(S)-2-Hydroxy-N,N-dimethylpentanamide ((S)-3c). HPLC (CHIRALPAK IC, $i$-PrOH/hexane $=1 / 4$, flow rate $=1.0 \mathrm{~mL} / \mathrm{min}): t \mathrm{R}=17.4 \mathrm{~min}(82.5 \%), t \mathrm{R}=36.2 \mathrm{~min}(17.5 \%) ; \mathrm{IR}$ (neat): $3425,1643 \mathrm{~cm}^{-1} ;{ }^{1} \mathrm{H} \mathrm{NMR}$ $\left(500 \mathrm{MHz}, \mathrm{CDCl}_{3}\right): \delta 4.26(\mathrm{~m}, 1 \mathrm{H}, 2-\mathrm{H}), 3.64(\mathrm{~d}, J=7.0 \mathrm{~Hz}, 1 \mathrm{H}, \mathrm{OH}), 2.90(\mathrm{~s}, 3 \mathrm{H}, \mathrm{NMe}), 2.89(\mathrm{~s}, 3 \mathrm{H}$, NMe), 1.52-1.47 (m, 1H, 3-H), 1.43-1.32 (m, 3H, 3-H, 4-H), 0.84 (dd, $J=7.5,7.5 \mathrm{~Hz}, 3 \mathrm{H}, 5-\mathrm{H}) ;{ }^{13} \mathrm{C}$ NMR $\left(125 \mathrm{MHz}, \mathrm{CDCl}_{3}\right): \delta 174.4,67.6,36.7,36.2,35.7,18.2,13.6$; HR MS: calcd for $\mathrm{C}_{7} \mathrm{H}_{15} \mathrm{NO}_{2} \mathrm{Na}[\mathrm{M}+\mathrm{Na}]^{+}$ 168.0995 , found 168.1000 .

(S)-2-Hydroxy-N,N,3-trimethylbutanamide ((S)-3d). HPLC (CHIRALPAK ID, $i$-PrOH $/$ hexane $=1 / 4$, flow rate $=1.0 \mathrm{~mL} / \mathrm{min}): t \mathrm{R}=8.7 \mathrm{~min}(42.8 \%), t \mathrm{R}=17.4 \mathrm{~min}(57.2 \%) ; \mathrm{IR}$ (neat): $3425,1643 \mathrm{~cm}^{-1} ;{ }^{1} \mathrm{H} \mathrm{NMR}$ $\left(500 \mathrm{MHz}, \mathrm{CDCl}_{3}\right): \delta 4.23(\mathrm{dd}, J=7.5,2.5 \mathrm{~Hz}, 1 \mathrm{H}, 2-\mathrm{H}), 3.56(\mathrm{~d}, J=7.0 \mathrm{~Hz}, 1 \mathrm{H}, \mathrm{OH}), 2.98(\mathrm{~s}, 3 \mathrm{H}, \mathrm{NMe})$, $2.97(\mathrm{~s}, 3 \mathrm{H}, \mathrm{NMe}), 1.91-1.82(\mathrm{~m}, 1 \mathrm{H}, 3-\mathrm{H}), 1.04(\mathrm{~d}, J=7.5 \mathrm{~Hz}, 3 \mathrm{H}, 4-\mathrm{H}), 0.77(\mathrm{~d}, J=7.5 \mathrm{~Hz}, 3 \mathrm{H}, 4-\mathrm{H}) ;{ }^{13} \mathrm{C}$ NMR $\left(125 \mathrm{MHz}, \mathrm{CDCl}_{3}\right): \delta 173.9,72.1,36.5,35.8,31.2,19.7,15.0$; HR MS: calcd for $\mathrm{C}_{7} \mathrm{H}_{15} \mathrm{NO}_{2} \mathrm{Na}[\mathrm{M}+$ $\mathrm{Na}]^{+}$168.0995, found 168.0994 .

(S)-2-Hydroxy-N,N-dimethylhexanamide ((S)-3e). HPLC (CHIRALPAK IC, $i$-PrOH $/$ hexane $=1 / 4$, flow rate $=1.0 \mathrm{~mL} / \mathrm{min}): t \mathrm{R}=13.7 \mathrm{~min}(84.7 \%), t \mathrm{R}=31.2 \mathrm{~min}(15.3 \%) ; \mathrm{IR}$ (neat): $3425,1643 \mathrm{~cm}^{-1} ;{ }^{1} \mathrm{H} \mathrm{NMR}$ $\left(500 \mathrm{MHz}, \mathrm{CDCl}_{3}\right): \delta 4.32(\mathrm{ddd}, J=7.5,7.5,3.5 \mathrm{~Hz}, 1 \mathrm{H}, 2-\mathrm{H}), 3.67(\mathrm{~d}, J=7.5 \mathrm{~Hz}, 1 \mathrm{H}, \mathrm{OH}), 2.98(\mathrm{~s}, 3 \mathrm{H}$, $\mathrm{NMe}), 2.96$ (s, 3H, NMe), 1.64-1.56 (m, 1H, 3-H), 1.48-1.24 (m, 5H, 3-H, 4-H, 5-H), 0.88 (dd, J = 7.5, 7.0 $\mathrm{Hz}, 3 \mathrm{H}, 6-\mathrm{H}) ;{ }^{13} \mathrm{C} \mathrm{NMR}\left(125 \mathrm{MHz}, \mathrm{CDCl}_{3}\right): \delta 174.5,67.9,36.3,35.8,34.3,27.1,22.4,13.9$; HR MS: calcd for $\mathrm{C}_{8} \mathrm{H}_{17} \mathrm{NO}_{2} \mathrm{Na}[\mathrm{M}+\mathrm{Na}]^{+}$182.1151, found 182.1149 .

(S)-2-Hydroxy-N,N,4-trimethylpentanamide ((S)-3f). HPLC (CHIRALPAK IC, $i$-PrOH $/$ hexane $=1 / 4$, flow rate $=1.0 \mathrm{~mL} / \mathrm{min}): t \mathrm{R}=14.6 \mathrm{~min}(89.2 \%), t \mathrm{R}=31.2 \mathrm{~min}(10.8 \%) ; \mathrm{IR}$ (neat): $3425,1642 \mathrm{~cm}^{-1} ;{ }^{1} \mathrm{H} \mathrm{NMR}$ 
$\left(500 \mathrm{MHz}, \mathrm{CDCl}_{3}\right): \delta 4.35$ (ddd, J = 7.0, $\left.2.5 \mathrm{~Hz}, 1 \mathrm{H}, 2-\mathrm{H}\right), 3.59$ (d, J = 7.0 Hz, $\left.1 \mathrm{H}, \mathrm{OH}\right), 2.96(\mathrm{~s}, 3 \mathrm{H}, \mathrm{NMe})$, $2.93(\mathrm{~s}, 3 \mathrm{H}, \mathrm{NMe}), 1.94(\mathrm{ddqq}, J=2.5,4.0,6.0,7.0 \mathrm{~Hz}, 1 \mathrm{H}, 4-\mathrm{H}), 1.38(\mathrm{ddd}, J=14.0,10.0,4.0 \mathrm{~Hz}, 1 \mathrm{H}, 3-\mathrm{H})$, $1.27(\mathrm{ddd}, J=14.0,10.0,2.5 \mathrm{~Hz}, 1 \mathrm{H}, 3-\mathrm{H}), 0.95(\mathrm{~d}, J=6.0 \mathrm{~Hz}, 3 \mathrm{H}, 5-\mathrm{H}), 0.91(\mathrm{~d}, J=7.0 \mathrm{~Hz}, 3 \mathrm{H}, 5-\mathrm{H}) ;{ }^{13} \mathrm{C}$ NMR (125 MHz, $\left.\mathrm{CDCl}_{3}\right): \delta 174.9,66.4,43.9,36.2,35.8,24.5,23.5,21.2 ;$ HR MS: calcd for $\mathrm{C}_{8} \mathrm{H}_{17} \mathrm{NO}_{2} \mathrm{Na}$ $[\mathrm{M}+\mathrm{Na}]^{+}$182.1151, found 182.1152 .

(S)-2-Cyclohexyl-2-Hydroxy-N,N-dimethylacetamide ((S)-3g). HPLC (CHIRALPAK IC, $i$-PrOH/hexane = $1 / 9$, flow rate $=1.0 \mathrm{~mL} / \mathrm{min}): t \mathrm{R}=11.0 \mathrm{~min}(53.2 \%), t \mathrm{R}=32.8 \mathrm{~min}(46.8 \%) ; \mathrm{IR}(\mathrm{KBr}): 3363,1628 \mathrm{~cm}^{-1}$; ${ }^{1} \mathrm{H}$ NMR (500 MHz, CDCl $): \delta 4.19$ (d, J = 1.5 Hz, 1H, 2-H), 3.54 (br s, 1H, OH), 2.98 (s, 3H, NMe), 2.97 (s, 3H, NMe), 1.77-1.72 (m, 2H, c-Hex), 1.62-1.60 (m, 2H, c-Hex), 1.50-1.37 (m, 3H, c-Hex), 1.26-1.05 (m, $4 \mathrm{H}, c-\mathrm{Hex}) ;{ }^{13} \mathrm{C}$ NMR $\left(125 \mathrm{MHz}, \mathrm{CDCl}_{3}\right): \delta 173.8,72.0,41.4,36.6,35.8,29.8,26.4,26.0,25.9,25.5 ; \mathrm{HR}$ MS: calcd for $\mathrm{C}_{10} \mathrm{H}_{19} \mathrm{NO}_{2} \mathrm{Na}[\mathrm{M}+\mathrm{Na}]^{+}$208.1308, found 208.1311.

(S)-3-(tert-Butyldimethylsiloxy)-2-hydroxy-N,N-dimethylpropanamide ((S)-3i). HPLC (CHIRALPAK IC, $i$-PrOH $/$ hexane $=1 / 9$, flow rate $=1.0 \mathrm{~mL} / \mathrm{min}): t \mathrm{R}=9.0 \mathrm{~min}(70.6 \%), t \mathrm{R}=13.3 \mathrm{~min}(29.4 \%) ; \mathrm{IR}$ (neat): 3278, $1635 \mathrm{~cm}^{-1}$; ${ }^{1} \mathrm{H}$ NMR (500 MHz, $\left.\mathrm{CDCl}_{3}\right): \delta 4.49$ (ddd, $\left.J=9.5,6.0,2.0 \mathrm{~Hz}, 1 \mathrm{H}, 2-\mathrm{H}\right), 3.78$ (dd, $J=10.0,5.0 \mathrm{~Hz}, 1 \mathrm{H}, 3-\mathrm{H}), 3.65(\mathrm{~d}, J=7.5 \mathrm{~Hz}, 1 \mathrm{H}, \mathrm{OH}), 3.63(\mathrm{dd}, J=10.0,7.5 \mathrm{~Hz}, 1 \mathrm{H}, 3-\mathrm{H}), 3.05(\mathrm{~s}, 3 \mathrm{H}$, $\mathrm{NMe}), 2.99$ (s, 3H, NMe), 0.86 (s, 9H, TBS), 0.04 (s, 3H, TBS), 0.03 (s, 3H, TBS); ${ }^{13} \mathrm{C}$ NMR $(125 \mathrm{MHz}$, $\mathrm{CDCl}_{3}$ ): $\delta 172.8,68.7,66.3,36.8,35.9,25.8,18.3,-5.5$; HR MS: calcd for $\mathrm{C}_{11} \mathrm{H}_{25} \mathrm{NO}_{3} \mathrm{SiNa}[\mathrm{M}+\mathrm{Na}]^{+}$ 270.1496 , found 270.1509 .

(S)-4-(tert-Butyldimethylsiloxy)-2-hydroxy-N,N-dimethylbutanamide ((S)-3j). HPLC (CHIRALPAK IC, $i$-PrOH $/$ hexane $=1 / 4$, flow rate $=1.0 \mathrm{~mL} / \mathrm{min}): t \mathrm{R}=11.4 \mathrm{~min}(3.5 \%), t \mathrm{R}=25.0 \mathrm{~min}(96.5 \%) ; \mathrm{R}$ (neat): $3363,1643 \mathrm{~cm}^{-1} ;{ }^{1} \mathrm{H}$ NMR $\left(500 \mathrm{MHz}, \mathrm{CDCl}_{3}\right): \delta 4.55-4.51(\mathrm{~m}, 1 \mathrm{H}, 2-\mathrm{H}), 3.85$ (ddd, $J=10.0,10.0,3.5 \mathrm{~Hz}$, $1 \mathrm{H}, 4-\mathrm{H}), 3.75(\mathrm{ddd}, J=10.0,10.0,3.5 \mathrm{~Hz}, 1 \mathrm{H}, 4-\mathrm{H}), 3.65$ (d, $J=7.5 \mathrm{~Hz}, 1 \mathrm{H}, \mathrm{OH}), 2.98$ (s, 6H, NMe), $1.85-1.80$ (m, 1H, 3-H), 1.58-1.51 (m, 1H, 3-H), 0.88 (s, 9H, TBS), 0.06 (s, 3H, TBS), 0.05 (s, 3H, TBS); ${ }^{13} \mathrm{C}$ NMR $\left(125 \mathrm{MHz}, \mathrm{CDCl}_{3}\right): \delta 174.6,64.9,59.2,38.3,36.1,35.8,25.8,18.2,-5.5$; HR MS: calcd for $\mathrm{C}_{12} \mathrm{H}_{27} \mathrm{NO}_{3} \mathrm{SiNa}[\mathrm{M}+\mathrm{Na}]^{+}$284.1652, found 284.1645.

(S)-5-(tert-Butyldimethylsiloxy)-2-hydroxy-N,N-dimethylpentanamide ((S)-3k). HPLC (CHIRALPAK IC, $i-\mathrm{PrOH} /$ hexane $=1 / 9$, flow rate $=1.0 \mathrm{~mL} / \mathrm{min}): t_{\mathrm{R}}=12.2 \mathrm{~min}(97.2 \%), t_{\mathrm{R}}=30.3 \mathrm{~min}(2.8 \%) ; \mathrm{IR}$ (neat): $3425,1643 \mathrm{~cm}^{-1} ;{ }^{1} \mathrm{H}$ NMR $\left(500 \mathrm{MHz}, \mathrm{CDCl}_{3}\right): \delta 4.36(\mathrm{~m}, 1 \mathrm{H}, 2-\mathrm{H}), 3.71(\mathrm{~d}, J=7.0 \mathrm{~Hz}, 1 \mathrm{H}, \mathrm{OH}), 3.69-3.59$ $(\mathrm{m}, 2 \mathrm{H}, 5-\mathrm{H}), 2.98$ (s, 3H, NMe), 2.96 (s, 3H. NMe), 1.80-1.73 (m, 1H, 3-H), 1.68-1.62 (m, 2H, 4-H), 1.52-1.44 (m, 1H, 3-H), 0.86 (s, 9H, TBS), 0.01 (s, 6H, TBS); ${ }^{13} \mathrm{C}$ NMR $\left(125 \mathrm{MHz}, \mathrm{CDCl}_{3}\right): \delta 174.4,67.6$, $62.3,36.3,35.8,30.9,28.0,25.8,18.2,-5.4$; HR MS: calcd for $\mathrm{C}_{13} \mathrm{H}_{29} \mathrm{NO}_{3} \mathrm{SiNa}[\mathrm{M}+\mathrm{Na}]^{+} 298.1809$ found 298.1805.

(R)-2-(Diphenylaceloxy)-N,N-dimethylpropanamide ((R)-4a). HPLC (CHIRALPAK IC, $i$-PrOH/hexane = $20 / 80$, flow rate $=1.0 \mathrm{~mL} / \mathrm{min}): t_{\mathrm{R}}=17.3 \mathrm{~min}(5.7 \%), t_{\mathrm{R}}=24.0 \mathrm{~min}(94.3 \%) ; \mathrm{IR}$ (neat): 1736, 1666, 1496, 1458, 741, $702 \mathrm{~cm}^{-1} ;{ }^{1} \mathrm{H}$ NMR (500 MHz, CDCl $): \delta 7.35-7.21(\mathrm{~m}, 10 \mathrm{H}, \mathrm{Ph}), 5.43(\mathrm{q}, J=6.0 \mathrm{~Hz}, 1 \mathrm{H}, 2-\mathrm{H})$, $5.12\left(\mathrm{~s}, 1 \mathrm{H}, 2^{\prime}-\mathrm{H}\right), 2.93\left(\mathrm{~s}, 6 \mathrm{H}, \mathrm{NMe}_{2}\right), 1.41(\mathrm{~d}, J=6.0 \mathrm{~Hz}, 3 \mathrm{H}, 3-\mathrm{H}) ;{ }^{13} \mathrm{C} \mathrm{NMR}\left(125 \mathrm{MHz}, \mathrm{CDCl}_{3}\right): \delta$ 172.1, 169.7, 138.4, 138.3, 128.7, 128.6, 128.5, 128.4, 127.2, 127.1, 67.7, 56.6, 36.6, 35.6, 16.5; HR MS: calcd for $\mathrm{C}_{19} \mathrm{H}_{21} \mathrm{NO}_{3} \mathrm{Na}[\mathrm{M}+\mathrm{Na}]^{+} 334.1414$, found 334.1407.

(R)-2-(Diphenylacetyloxy)-N,N-dimethylbutanamide ((R)-4b). HPLC (CHIRALPAK IC, $i-\mathrm{PrOH} /$ hexane $=$ $20 / 80$, flow rate $=1.0 \mathrm{~mL} / \mathrm{min}): t_{R}=15.6 \mathrm{~min}(3.7 \%), t_{\mathrm{R}}=28.2 \mathrm{~min}(96.3 \%) ; \mathrm{IR}$ (neat): 1736, 1658, 1496, 1458, 741, $702 \mathrm{~cm}^{-1} ;{ }^{1} \mathrm{H}$ NMR (500 MHz, $\left.\mathrm{CDCl}_{3}\right): \delta 7.34-7.18(\mathrm{~m}, 10 \mathrm{H}, \mathrm{Ph}), 5.21(\mathrm{dd}, J=7.5,5.5 \mathrm{~Hz}, 1 \mathrm{H}$, 2-H), $5.11\left(\mathrm{~s}, 1 \mathrm{H}, 2^{\prime}-\mathrm{H}\right), 2.96(\mathrm{~s}, 3 \mathrm{H}, \mathrm{NMe}), 2.91(\mathrm{~s}, 3 \mathrm{H}, \mathrm{NMe}) 1.79-1.71(\mathrm{~m}, 2 \mathrm{H}, 3-\mathrm{H}), 0.84(\mathrm{t}, J=7.5 \mathrm{~Hz}$, $3 \mathrm{H}, 4-\mathrm{H}) ;{ }^{13} \mathrm{C} \mathrm{NMR}\left(125 \mathrm{MHz}, \mathrm{CDCl}_{3}\right): \delta 172.4,169.3,138.5,128.7,128.6,128.5,128.4,127.2,127.1,72.5$, 56.7, 36.7, 35.8, 24.3, 9.6; HR MS: calcd for $\mathrm{C}_{20} \mathrm{H}_{23} \mathrm{NO}_{3} \mathrm{Na}[\mathrm{M}+\mathrm{Na}]^{+} 348.1570$, found 348.1577. 
(R)-2-(Diphenylacetyloxy)-N,N-dimethylpentanamide ((R)-4c). HPLC (CHIRALPAK IC, $i$-PrOH $/$ hexane = $20 / 80$, flow rate $=1.0 \mathrm{~mL} / \mathrm{min}): t_{\mathrm{R}}=13.8 \mathrm{~min}(2.9 \%), t_{\mathrm{R}}=27.6 \mathrm{~min}(97.1 \%) ; \mathrm{IR}$ (neat): 1736, 1666, 1496, 1458, 741, $702 \mathrm{~cm}^{-1},{ }^{1} \mathrm{H}$ NMR (500 MHz, $\left.\mathrm{CDCl}_{3}\right): \delta 7.36-7.21(\mathrm{~m}, 10 \mathrm{H}, \mathrm{Ph}), 5.31(\mathrm{dd}, J=8.5,4.5 \mathrm{~Hz}, 1 \mathrm{H}$, 2-H), 5.14 (s, 1H, 2'-H), 3.00 (s, 3H, NMe), 2.94 (s, 3H, NMe), 1.82-1.76 (m, 1H, 3-H), 1.70-1.64 (m, 1H, 3-H), 1.38-1.22 (m, 2H, 4-H), 0.85 (t, $J=7.5 \mathrm{~Hz}, 3 \mathrm{H}, 5-\mathrm{H}) ;{ }^{13} \mathrm{C}$ NMR $\left(125 \mathrm{MHz}, \mathrm{CDCl}_{3}\right): \delta 172.4,169.5$, $138.5,138.5,128.7,128.6,128.5,128.4,127.2,127.1,71.0,56.6,36.7,35.9,32.9,18.4,13.5$; HR MS: calcd for $\mathrm{C}_{21} \mathrm{H}_{25} \mathrm{NO}_{3} \mathrm{Na}[\mathrm{M}+\mathrm{Na}]^{+}$362.1727, found 362.1733 .

(R)-2-(Diphenylacetyloxy)-N,N,3-trimethylbutanamide ((R)-4d). HPLC (CHIRALPAK ID, $i$-PrOH/hexane $=20 / 80$, flow rate $=1.0 \mathrm{~mL} / \mathrm{min}): t_{\mathrm{R}}=8.7 \mathrm{~min}(90.9 \%), t_{\mathrm{R}}=18.4 \mathrm{~min}(9.1 \%) ; \mathrm{IR}$ (neat): 1736, 1658, 1496, 1458, 748, $702 \mathrm{~cm}^{-1} ;{ }^{1} \mathrm{H}$ NMR (500 MHz, $\left.\mathrm{CDCl}_{3}\right): \delta 7.31-7.14(\mathrm{~m}, 10 \mathrm{H}, \mathrm{Ph}), 5.08\left(\mathrm{~s}, 1 \mathrm{H}, 2^{\prime}-\mathrm{H}\right), 4.99(\mathrm{~d}$, $J=7.5 \mathrm{~Hz}, 1 \mathrm{H}, 2-\mathrm{H}), 3.00$ (s, 3H, NMe), 2.89 (s, 3H, NMe), 2.08 (m, 1H, 3-H), 0.80 (d, J = 7.5 Hz, 3H, $4-\mathrm{H}), 0.78(\mathrm{~d}, J=6.0 \mathrm{~Hz}, 3 \mathrm{H}, 4-\mathrm{H}) ;{ }^{13} \mathrm{C} \mathrm{NMR}\left(125 \mathrm{MHz}, \mathrm{CDCl}_{3}\right): \delta 172.5,169.1,138.5,138.5,128.7,128.7$, $128.5,128.3,127.2,127.1,75.6,56.8,37.0,35.9,30.1,18.4,17.7$; HR MS: calcd for $\mathrm{C}_{21} \mathrm{H}_{25} \mathrm{NO}_{3} \mathrm{Na}[\mathrm{M}+$ $\mathrm{Na}]^{+}$362.1727, found 362.1710 .

(R)-2-(Diphenylacetyloxy)-N,N-dimethylhexanamide ((R)-4e). HPLC (CHIRALPAK IC, $i$-PrOH/hexane $=20 / 80$, flow rate $=1.0 \mathrm{~mL} / \mathrm{min}): t_{\mathrm{R}}=13.4 \mathrm{~min}(2.7 \%), t_{\mathrm{R}}=30.0 \mathrm{~min}(97.3 \%) ; \mathrm{IR}($ neat $): 1736,1666$, 1496, 1458, 741, $702 \mathrm{~cm}^{-1} ;{ }^{1} \mathrm{H}$ NMR (500 MHz, $\left.\mathrm{CDCl}_{3}\right): \delta 7.37-7.21(\mathrm{~m}, 10 \mathrm{H}, \mathrm{Ph}), 5.30(\mathrm{dd}, J=5.0,5.0$ $\mathrm{Hz}, 1 \mathrm{H}, 2-\mathrm{H}), 5.14$ (s, 1H, 2'-H), 3.00 (s, 3H, NMe), 2.94 (s, 3H, NMe), 1.80 (m, 1H, 3-H), 1.70 (m, 1H, 3-H), 1.27-1.20 (m, 4H, 4-H, 5-H), 0.77 (t, $J=6.5,6.0 \mathrm{~Hz}, 3 \mathrm{H}, 3-\mathrm{H}) ;{ }^{13} \mathrm{C} \mathrm{NMR}\left(125 \mathrm{MHz}, \mathrm{CDCl}_{3}\right): \delta 172.4$, 169.6, 138.5, 138.5, 128.7, 128.7, 128.6, 128.4, 127.2, 127.1, 71.2, 56.7, 36.7, 35.9, 30.6, 27.2, 22.1, 13.7; HR MS: calcd for $\mathrm{C}_{22} \mathrm{H}_{27} \mathrm{NO}_{3} \mathrm{Na}[\mathrm{M}+\mathrm{Na}]^{+} 376.1883$, found 376.1898.

(R)-2-(Diphenylacetyloxy)-N,N,4-trimethylpentanamide ((R)-4f). HPLC (CHIRALPAK IC, $i$-PrOH/hexane $=20 / 80$, flow rate $=1.0 \mathrm{~mL} / \mathrm{min}): t_{\mathrm{R}}=14.8 \mathrm{~min}(2.5 \%), t_{\mathrm{R}}=31.3 \mathrm{~min}(97.5 \%)$; IR (neat): 1736, 1666, 1496, 1458, 741, $702 \mathrm{~cm}^{-1} ;{ }^{1} \mathrm{H}$ NMR (500 MHz, $\left.\mathrm{CDCl}_{3}\right): \delta 7.33-7.18(\mathrm{~m}, 10 \mathrm{H}, \mathrm{Ph}), 5.32(\mathrm{dd}, J=10.4,3.4$ $\mathrm{Hz}, 1 \mathrm{H}, 2-\mathrm{H}), 5.12$ (s, 1H, 2'-H), 2.98 (s, 3H, NMe), 2.91 (s, 3H, NMe), 1.79 (ddd, J = 14.6, 10.4, 4.6, 1H, 3-H), 1.54-1.51 (m, 1H, 4-H), 1.38 (ddd, $J=14.0,9.2,3.4$ Hz, 1H, 3-H), 0.81 (d, J = 6.7 Hz, 3H, 5-H), 0.79 $(\mathrm{d}, J=6.4 \mathrm{~Hz}, 3 \mathrm{H}, 5-\mathrm{H}) ;{ }^{13} \mathrm{C} \mathrm{NMR}\left(125 \mathrm{MHz}, \mathrm{CDCl}_{3}\right): \delta 172.5,169.8,138.5,138.4,128.7,128.7,128.5$, $128.4,127.2,127.1,69.9,56.7,39.6,36.6,35.9,24.4,23.0,21.3$; HR MS: calcd for $\mathrm{C}_{22} \mathrm{H}_{27} \mathrm{NO}_{3} \mathrm{Na}[\mathrm{M}+\mathrm{Na}]^{+}$ 376.1883 , found 376.1873 .

(R)-2-Cyclohexyl-2-(diphenylacetyloxy)-N,N-dimethylacetamide ((R)-4g). HPLC (CHIRALPAK IC, $i-\mathrm{PrOH} /$ hexane $=40 / 60$, flow rate $=0.75 \mathrm{~mL} / \mathrm{min}): t_{\mathrm{R}}=11.2 \mathrm{~min}(16.8 \%), t_{\mathrm{R}}=31.3 \mathrm{~min}(83.2 \%)$; IR (neat): 1736, 1658, 1496, 1450, 748, $702 \mathrm{~cm}^{-1} ;{ }^{1} \mathrm{H}$ NMR (500 MHz, $\left.\mathrm{CDCl}_{3}\right): \delta 7.29-7.13(\mathrm{~m}, 10 \mathrm{H}, \mathrm{Ph})$, 5.06 (s, 1H, 2'-H), 5.00 (d, J = 7.3 Hz, 1H, 2-H), 3.00 (s, 3H, NMe), 2.88 (s, 3H, NMe), 1.77 (m, 1H, 3-H), 1.58-1.45 (m, 5H, c-Hex), 1.17-0.80 (m, 5H, c-Hex); $\left.{ }^{13} \mathrm{C} \mathrm{NMR} \mathrm{(125} \mathrm{MHz,} \mathrm{CDCl}_{3}\right): \delta 172.5,169.1,138.6$, $138.5,128.8,128.8,128.6,128.4,127.2,127.1,75.0,56.7,39.5,37.1,35.9,28.5,28.2,26.0,25.8,25.5$; HR MS: calcd for $\mathrm{C}_{24} \mathrm{H}_{29} \mathrm{NO}_{3} \mathrm{Na}[\mathrm{M}+\mathrm{Na}]^{+} 402.2040$, found 402.2047 .

(R)-3-(tert-Butyldimethylsiloxy)-2-(diphenylacetyloxy)-N,N-dimethylpropanamide ((R)-4i). HPLC (CHIRALPAK $\mathrm{IC}, i-\mathrm{PrOH} /$ hexane $=20 / 80$, flow rate $=1.0 \mathrm{~mL} / \mathrm{min}): t_{\mathrm{R}}=11.4 \mathrm{~min}(9.0 \%), t_{\mathrm{R}}=13.3 \mathrm{~min}(91.0 \%) ; \mathrm{IR}$ (neat): 1743, 1658, 1496, 1458, 741, $702 \mathrm{~cm}^{-1} ;{ }^{1} \mathrm{H}$ NMR (500 MHz, CDCl $): \delta 7.36-7.23$ (m, 10H, Ph), $5.49(\mathrm{t}, J=6.0 \mathrm{~Hz}, 1 \mathrm{H}, 2-\mathrm{H}), 5.15\left(\mathrm{~s}, 1 \mathrm{H}, 2^{\prime}-\mathrm{H}\right), 3.90(\mathrm{~m}, 2 \mathrm{H}, 3-\mathrm{H}), 3.10(\mathrm{~s}, 3 \mathrm{H}, \mathrm{NMe}), 2.97$ (s, 3H, NMe), 0.85 (s, 9H, TBS), 0.02 (s, 3H, TBS), 0.00 (s, 3H, TBS); ${ }^{13} \mathrm{C} \mathrm{NMR} \mathrm{(125} \mathrm{MHz,} \mathrm{CDCl}_{3}$ ): $\delta 172.2,168.1,138.4$, 138.4, 128.7, 128.6, 128.5, 127.3, 127.2, 71.6, 62.9, 56.6, 37.0, 36.0, 25.7, 18.1, 5.6, 5.7; HR MS: calcd for $\mathrm{C}_{25} \mathrm{H}_{35} \mathrm{NO}_{4} \mathrm{SiNa}[\mathrm{M}+\mathrm{Na}]^{+} 464.2228$, found 464.2222 .

(R)-4-(tert-Butyldimethylsiloxy)-2-(diphenylacetyloxy)-N,N-dimethylbutanamide ((R)-4j). HPLC (CHIRALPAK $\mathrm{IC}, i-\mathrm{PrOH} /$ hexane $=20 / 80$, flow rate $=1.0 \mathrm{~mL} / \mathrm{min}): t_{\mathrm{R}}=11.4 \mathrm{~min}(3.5 \%), t_{\mathrm{R}}=25.0 \mathrm{~min}(96.5 \%) ; \mathrm{IR}$ 
(neat): 1743, 1666, 1496, 1466, 748, $717 \mathrm{~cm}^{-1} ;{ }^{1} \mathrm{H}$ NMR (500 MHz, $\left.\mathrm{CDCl}_{3}\right): \delta 7.38-7.22(\mathrm{~m}, 10 \mathrm{H}, \mathrm{Ph})$, $5.54(\mathrm{dd}, J=9.5,3.0 \mathrm{~Hz}, 1 \mathrm{H}, 2-\mathrm{H}), 5.17\left(\mathrm{~s}, 1 \mathrm{H}, 2^{\prime}-\mathrm{H}\right), 3.60(\mathrm{dt}, J=10.0,5.0 \mathrm{~Hz}, 1 \mathrm{H}, 4-\mathrm{H}), 3.48(\mathrm{dt}, J=10.0$, $3.5 \mathrm{~Hz}, 1 \mathrm{H}, 4-\mathrm{H}), 3.08$ (s, 3H, NMe), $2.98(\mathrm{~s}, 3 \mathrm{H}, \mathrm{NMe}), 1.98(\mathrm{~m}, 1 \mathrm{H}, 3-\mathrm{H}), 1.89(\mathrm{~m}, 1 \mathrm{H}, 3-\mathrm{H}) 0.86(\mathrm{~s}, 9 \mathrm{H}$, TBS), 0.02 (s, 3H, TBS), 0.06 (s, 3H, TBS); ${ }^{13} \mathrm{C}$ NMR $\left(125 \mathrm{MHz}, \mathrm{CDCl}_{3}\right): \delta 172.3,169.8,138.6,138.5,128.8$, 128.7, 128.6, 128.4, 127.2, 127.1, 68.0, 58.4, 56.7, 36.6, 35.8, 34.2, 25.8, 18.1, 5.6, 5.7; HR MS: calcd for $\mathrm{C}_{26} \mathrm{H}_{37} \mathrm{NO}_{4} \mathrm{SiNa}[\mathrm{M}+\mathrm{Na}]^{+} 478.2384$, found 478.2386 .

(R)-5-(tert-Butyldimethylsiloxy)-2-(Diphenylacetyloxy)-N,N-dimethylpentanamide ((R)-4k). HPLC (CHIRALPAK $\mathrm{IC}, i-\mathrm{PrOH} /$ hexane $=20 / 80$, flow rate $=1.0 \mathrm{~mL} / \mathrm{min}): t_{\mathrm{R}}=12.2 \mathrm{~min}(2.4 \%), t_{\mathrm{R}}=29.7 \mathrm{~min}(97.6 \%) ; \mathrm{IR}$ (neat): 1751, 1666, 1496, 1458, 748, $702 \mathrm{~cm}^{-1},{ }^{1} \mathrm{H}$ NMR (500 MHz, $\left.\mathrm{CDCl}_{3}\right): \delta 7.37-7.22(\mathrm{~m}, 10 \mathrm{H}, \mathrm{Ph})$, $5.35(\mathrm{t}, J=6.5 \mathrm{~Hz}, 1 \mathrm{H}, 2-\mathrm{H}), 5.15\left(\mathrm{~s}, 1 \mathrm{H}, 2^{\prime}-\mathrm{H}\right), 3.54(\mathrm{t}, J=6.0 \mathrm{~Hz}, 2 \mathrm{H}, 5-\mathrm{H}), 3.02(\mathrm{~s}, 3 \mathrm{H}, \mathrm{NMe}), 2.95(\mathrm{~s}, 3 \mathrm{H}$, $\mathrm{NMe}), 1.84(\mathrm{dt}, J=6.5,6.5 \mathrm{~Hz}, 2 \mathrm{H}, 3-\mathrm{H}), 1.55-1.39$ (m, 2H, 4-H), 0.87 (s, 9H, TBS), 0.01 (s, 6H, TBS); ${ }^{13} \mathrm{C}$ NMR (125 MHz, $\mathrm{CDCl}_{3}$ ): $\delta 172.4,169.5,138.5,138.5,128.8,128.7,128.6,128.4,127.2,127.1,71.1,62.0$, 56.7, 36.7, 35.9, 28.0, 27.3, 25.9, 18.2, 5.4; HR MS: calcd for $\mathrm{C}_{27} \mathrm{H}_{39} \mathrm{NO}_{4} \mathrm{SiNa}[\mathrm{M}+\mathrm{Na}]^{+} 492.2541$, found 492.2554 .

(S)-2-Hydroxy-N-methoxy-N-methylpropanamide ((S)-5a). HPLC (CHIRALPAK IC, $i$-PrOH/hexane = 1/9, flow rate $=0.5 \mathrm{~mL} / \mathrm{min}): t_{\mathrm{R}}=16.6 \mathrm{~min}(99.2 \%), t_{\mathrm{R}}=27.3 \mathrm{~min}(0.8 \%) ; \mathrm{IR}$ (neat): $3443,1662 \mathrm{~cm}^{-1} ;{ }^{1} \mathrm{H}$ NMR $\left(\mathrm{CDCl}_{3}\right): \delta 4.42(\mathrm{dq}, J=7.0,7.0 \mathrm{~Hz}, 1 \mathrm{H}, 2-\mathrm{H}), 3.65(\mathrm{~s}, 3 \mathrm{H}, \mathrm{OMe}), 3.42(\mathrm{~d}, J=7.0 \mathrm{~Hz}, 1 \mathrm{H}, \mathrm{OH}), 2.81$ (s, 3H, NMe), 1.29 (d, J = 7.0 Hz, 3H, 3-H); ${ }^{13} \mathrm{C} \mathrm{NMR}\left(\mathrm{CDCl}_{3}\right): \delta 175.6,64.8,61.1,32.2,20.8$; HR MS: calcd for $\mathrm{C}_{5} \mathrm{H}_{11} \mathrm{NO}_{3} \mathrm{Na}[\mathrm{M}+\mathrm{Na}]^{+}$156.0631, found 156.0634 .

(S)-2-Hydroxy-N-methoxy-N-methylbutanamide ((S)-5b). HPLC (CHIRALPAK IC, $i$-PrOH/hexane = 1/9, flow rate $=1.0 \mathrm{~mL} / \mathrm{min}): t_{\mathrm{R}}=13.6 \mathrm{~min}(92.4 \%), t_{\mathrm{R}}=41.3 \min (7.6 \%) ; \mathrm{IR}$ (neat): $3448,1658 \mathrm{~cm}^{-1} ;{ }^{1} \mathrm{H}$ $\operatorname{NMR}\left(\mathrm{CDCl}_{3}\right): \delta 4.33(\mathrm{ddd}, J=7.5,7.5,3.5 \mathrm{~Hz}, 1 \mathrm{H}, 2-\mathrm{H}), 3.69(\mathrm{~s}, 3 \mathrm{H}, \mathrm{OMe}), 3.24(\mathrm{~d}, J=7.5 \mathrm{~Hz}, 1 \mathrm{H}, \mathrm{OH})$, $3.22(\mathrm{~s}, 3 \mathrm{H}, \mathrm{NMe}), 1.76(\mathrm{dqd}, J=14.5,7.5,3.5 \mathrm{~Hz}, 1 \mathrm{H}, 3-\mathrm{H}), 1.55(\mathrm{ddq}, J=14.5,7.5,7.5 \mathrm{~Hz}, 1 \mathrm{H}, 3-\mathrm{H})$, $0.95(\mathrm{dd}, J=7.5,7.5 \mathrm{~Hz}, 3 \mathrm{H}, 4-\mathrm{H}) ;{ }^{13} \mathrm{C} \mathrm{NMR}\left(\mathrm{CDCl}_{3}\right): \delta 175.0,69.6,61.2,32.3,27.6,9.1$; HR MS: calcd for $\mathrm{C}_{6} \mathrm{H}_{13} \mathrm{NO}_{3} \mathrm{Na}[\mathrm{M}+\mathrm{Na}]^{+} 170.0788$, found 170.0793 .

(S)-2-Hydroxy-N-methoxy-N-methylpentanamide ((S)-5c). HPLC (CHIRALPAK IC, $i$-PrOH/hexane = 1/9, flow rate $=1.0 \mathrm{~mL} / \mathrm{min}): t_{\mathrm{R}}=13.1 \mathrm{~min}(96.8 \%), t_{\mathrm{R}}=33.4 \mathrm{~min}(3.2 \%) ; \mathrm{IR}$ (neat): $3464,1658 \mathrm{~cm}^{-1} ;{ }^{1} \mathrm{H}$ NMR $\left(\mathrm{CDCl}_{3}\right): \delta 4.43-4.24(\mathrm{~m}, 1 \mathrm{H}, 2-\mathrm{H}), 3.66(\mathrm{dd}, J=14.0,14.0 \mathrm{~Hz}, 3 \mathrm{H}, \mathrm{OMe}), 3.28-3.20(\mathrm{~m}, 1 \mathrm{H}, \mathrm{OH})$, $3.19(\mathrm{dd}, J=14.0,14.0 \mathrm{~Hz}, 3 \mathrm{H}, \mathrm{NMe}), 1.71-1.57(\mathrm{~m}, 1 \mathrm{H}, 3-\mathrm{H}), 1.53-1.35(\mathrm{~m}, 3 \mathrm{H}, 3-\mathrm{H}, 4-\mathrm{H}), 0.89$ (dddd, $J$ $=15.0,15.0,7.5,7.5 \mathrm{~Hz}, 3 \mathrm{H}, 5-\mathrm{H}) ;{ }^{13} \mathrm{C} \mathrm{NMR}\left(\mathrm{CDCl}_{3}\right): \delta 175.2,68.3,61.1,36.8,36.7,32.3,18.2,18.1,13.6$; HR MS: calcd for $\mathrm{C}_{7} \mathrm{H}_{15} \mathrm{NO}_{3} \mathrm{Na}[\mathrm{M}+\mathrm{Na}]^{+} 184.0944$, found 184.0941 .

(S)-2-Hydroxy-N-methoxy-N,3-dimethylbutanamide ((S)-5d). HPLC (CHIRALPAK IC, $i$-PrOH $/$ hexane $=$ $1 / 9$, flow rate $=1.0 \mathrm{~mL} / \mathrm{min}): t_{\mathrm{R}}=11.1 \mathrm{~min}(53.4 \%), t_{\mathrm{R}}=31.8 \min (46.6 \%) ; \mathrm{IR}($ neat $): 3455,1656 \mathrm{~cm}^{-1}$; ${ }^{1} \mathrm{H}$ NMR $\left(\mathrm{CDCl}_{3}\right): \delta 4.23(\mathrm{dd}, J=8.0,2.5 \mathrm{~Hz}, 1 \mathrm{H}, 2-\mathrm{H}), 3.67(\mathrm{~s}, 3 \mathrm{H}, \mathrm{OMe}), 3.21(\mathrm{~s}, 3 \mathrm{H}, \mathrm{NMe}), 3.13(\mathrm{~d}, J=$ $8.0 \mathrm{~Hz}, 1 \mathrm{H}, \mathrm{OH}), 2.05-1.93(\mathrm{~m}, 1 \mathrm{H}, 3-\mathrm{H}), 1.00(\mathrm{~d}, J=7.0 \mathrm{~Hz}, 3 \mathrm{H}, 4-\mathrm{H}), 0.78(\mathrm{~d}, J=7.0 \mathrm{~Hz}, 3 \mathrm{H}, 4-\mathrm{H}) ;{ }^{13} \mathrm{C}$ $\operatorname{NMR}\left(\mathrm{CDCl}_{3}\right): \delta 174.6,72.8,32.3,31.3,19.6,15.2 ; \mathrm{HR}$ MS: calcd for $\mathrm{C}_{7} \mathrm{H}_{15} \mathrm{NO}_{3} \mathrm{Na}[\mathrm{M}+\mathrm{Na}]^{+}$184.0944, found 184.0949 .

(S)-2-Hydroxy-N-methoxy-N-methylhexanamide ((S)-5e). HPLC (CHIRALPAK IC, $i$-PrOH/hexane = 1/9, flow rate $=1.0 \mathrm{~mL} / \mathrm{min}): t_{\mathrm{R}}=10.3 \mathrm{~min}(84.3 \%), t_{\mathrm{R}}=26.1 \mathrm{~min}(15.7 \%) ; \mathrm{IR}$ (neat): $3449,1658 \mathrm{~cm}^{-1} ;{ }^{1} \mathrm{H}$ NMR $\left(\mathrm{CDCl}_{3}\right): \delta 4.37-4.34(\mathrm{~m}, 1 \mathrm{H}, 2-\mathrm{H}), 3.68(\mathrm{~s}, 3 \mathrm{H}, \mathrm{OMe}), 3.23(\mathrm{~s}, 1 \mathrm{H}, \mathrm{OH}), 3.21(\mathrm{~s}, 3 \mathrm{H}, \mathrm{NMe}), 1.75-1.64$ $(\mathrm{m}, 1 \mathrm{H}, 3-\mathrm{H}), 1.55-1.21(\mathrm{~m}, 3 \mathrm{H}, 3-\mathrm{H}, 4-\mathrm{H}), 0.88(\mathrm{dd}, J=7.5,7.5 \mathrm{~Hz}, 3 \mathrm{H}, 5-\mathrm{H}) ;{ }^{13} \mathrm{C} \mathrm{NMR}\left(\mathrm{CDCl}_{3}\right): \delta$ 175.3, 68.6, 61.2, 34.3, 32.3, 27.0, 22.3, 13.8; HR MS: calcd for $\mathrm{C}_{8} \mathrm{H}_{17} \mathrm{NO}_{3} \mathrm{Na}[\mathrm{M}+\mathrm{Na}]^{+}$198.1101, found 198.1110 . 
(S)-2-Hydroxy-N-methoxy-N,4-dimethylpentanamide ((S)-5f). HPLC (CHIRALPAK IC, $i$-PrOH/hexane = $1 / 9$, flow rate $=0.5 \mathrm{~mL} / \mathrm{min}): t_{\mathrm{R}}=20.0 \mathrm{~min}(82.3 \%), t_{\mathrm{R}}=50.9 \mathrm{~min}(4.8 \%) ; \mathrm{IR}($ neat $): 3447,1660 \mathrm{~cm}^{-1}$; ${ }^{1} \mathrm{H} \mathrm{NMR}\left(\mathrm{CDCl}_{3}\right): \delta 4.39(\mathrm{dd}, J=8.0,8.0 \mathrm{~Hz}, 1 \mathrm{H}, 2-\mathrm{H}), 3.68(\mathrm{~s}, 3 \mathrm{H}, \mathrm{OMe}), 3.20(\mathrm{~s}, 3 \mathrm{H}, \mathrm{NMe}), 3.15(\mathrm{~d}$, $J=8.0 \mathrm{~Hz}, 1 \mathrm{H}, \mathrm{OH}), 1.95-1.84(\mathrm{~m}, 1 \mathrm{H}, 4-\mathrm{H}), 1.48-1.33(\mathrm{~m}, 2 \mathrm{H}, 3-\mathrm{H}), 0.93(\mathrm{~d}, J=7.0 \mathrm{~Hz}, 3 \mathrm{H}, 5-\mathrm{H}), 0.91(\mathrm{~d}$, $J=6.5 \mathrm{~Hz}, 3 \mathrm{H}, 5-\mathrm{H}) ;{ }^{13} \mathrm{C} \mathrm{NMR}\left(\mathrm{CDCl}_{3}\right): \delta 175.7,67.2,61.1,43.9,32.4,24.5,23.5,21.2 ; \mathrm{HR} \mathrm{MS}$ : calcd for $\mathrm{C}_{8} \mathrm{H}_{17} \mathrm{NO}_{3} \mathrm{Na}[\mathrm{M}+\mathrm{Na}]^{+}$198.1101, found 198.1097 .

(S)-2-Cyclohexyl-2-hydroxy-N-methoxy-N-methylacetamide ((S)-5h). HPLC (CHIRALPAK IC, $i$-PrOH/ hexane $=1 / 9$, flow rate $=1.0 \mathrm{~mL} / \mathrm{min}): t_{\mathrm{R}}=10.2 \mathrm{~min}(52.5 \%), t_{\mathrm{R}}=40.4 \mathrm{~min}(47.5 \%) ; \mathrm{IR}($ neat $): 3451$, $1656 \mathrm{~cm}^{-1} ;{ }^{1} \mathrm{H}$ NMR $\left(\mathrm{CDCl}_{3}\right): \delta 4.20(\mathrm{~d}, J=4.0 \mathrm{~Hz}, 1 \mathrm{H}, 2-\mathrm{H}), 3.67$ (s, 3H, OMe), $3.21(\mathrm{~s}, 3 \mathrm{H}, \mathrm{NMe}), 3.13$ $(\mathrm{d}, J=8.0 \mathrm{~Hz}, 1 \mathrm{H}, \mathrm{OH}), 1.76-1.53(\mathrm{~m}, 5 \mathrm{H}, c-\mathrm{Hex}), 1.47-1.30$ (m, 2H, $c$-Hex), 1.26-1.03 (m, 4H, $c-\mathrm{Hex})$; ${ }^{13} \mathrm{C} \mathrm{NMR}\left(\mathrm{CDCl}_{3}\right): \delta 174.4,72.6,61.1,41.4,32.2,29.6,26.3,26.0,25.9 ; \mathrm{HR}$ MS: calcd for $\mathrm{C}_{10} \mathrm{H}_{19} \mathrm{NO}_{3} \mathrm{Na}$ $[\mathrm{M}+\mathrm{Na}]^{+} 224.1257$, found 224.1248 .

(S)-3-(tert-Butyldimethylsiloxy)-2-hydroxy-N-methoxy-N-methylpropanamide ((S)-5i). HPLC (CHIRALPAK $\mathrm{IC}, i-\mathrm{PrOH} /$ hexane $=1 / 9$, flow rate $=0.5 \mathrm{~mL} / \mathrm{min}): t_{\mathrm{R}}=13.7 \mathrm{~min}(75.4 \%), t_{\mathrm{R}}=20.2 \mathrm{~min}(24.6 \%) ; \mathrm{IR}$ (neat): 3447, $1665 \mathrm{~cm}^{-1} ;{ }^{1} \mathrm{H}$ NMR $\left(\mathrm{CDCl}_{3}\right): \delta 4.52-4.35(\mathrm{~m}, 1 \mathrm{H}, 2-\mathrm{H}), 3.86(\mathrm{dd}, J=10.0,3.5 \mathrm{~Hz}, 1 \mathrm{H}, 3-\mathrm{H})$, $3.81(\mathrm{dd}, J=10.0,3.5 \mathrm{~Hz}, 1 \mathrm{H}, 3-\mathrm{H}), 3.70$ (dd, $J=15.0,15.0 \mathrm{~Hz}, 3 \mathrm{H}, \mathrm{OMe}), 3.48$ (ddd, $J=15.0,15.0,8.5 \mathrm{~Hz}$, 1H, OH), 3.23 (dd, $J=15.0,15.0 \mathrm{~Hz}, 3 \mathrm{H}, \mathrm{NMe}), 0.86$ (dd, $J=15.0,15.0 \mathrm{~Hz}, 9 \mathrm{H}, \mathrm{TBS}), 0.04$ (dd, $J=15.0$, $15.0 \mathrm{~Hz}, 3 \mathrm{H}, \mathrm{TBS}), 0.03$ (s, 3H, TBS); ${ }^{13} \mathrm{C} \mathrm{NMR}\left(\mathrm{CDCl}_{3}\right): \delta 172.3,70.2,65.2,61.2,32.4,25.8,18.3,-5.4$, -5.5; HR MS: calcd for $\mathrm{C}_{11} \mathrm{H}_{25} \mathrm{NO}_{4} \mathrm{SiNa}[\mathrm{M}+\mathrm{Na}]^{+} 286.1445$, found 286.1431.

(S)-4-(tert-Butyldimethylsiloxy)-2-hydroxy-N-methoxy-N-methylbutanamide ((S)-5j). HPLC (CHIRALPAK $\mathrm{IC}, i-\mathrm{PrOH} /$ hexane $=1 / 9$, flow rate $=1.0 \mathrm{~mL} / \mathrm{min}): t_{\mathrm{R}}=9.6 \mathrm{~min}(95.2 \%), t_{\mathrm{R}}=23.1 \mathrm{~min}(4.8 \%)$; IR (neat): 3451, 1662, $\mathrm{cm}^{-1},{ }^{1} \mathrm{H}$ NMR $\left(\mathrm{CDCl}_{3}\right): \delta 4.62-4.48(\mathrm{~m}, 1 \mathrm{H}, 2-\mathrm{H}), 3.90-3.74(\mathrm{~m}, 2 \mathrm{H}, 4-\mathrm{H}), 3.70(\mathrm{dd}, \mathrm{J}=15.0$, $15.0 \mathrm{~Hz}, 3 \mathrm{H}, \mathrm{OMe}$ ), 3.28 (d, $J=7.0 \mathrm{~Hz}, 1 \mathrm{H}, \mathrm{OH}), 3.23$ (ddd, $J=14.5,14.5,5.0 \mathrm{~Hz}, 3 \mathrm{H}, \mathrm{NMe}), 2.05-1.88$ (m, 1H, 3-H), 1.68-1.55 (m, 1H, 3-H), 0.89 (dd, $J=15.0,15.0$ Hz, 9H, TBS), 0.06 (ddd, $J=14.5,14.5,5.0$ $\mathrm{Hz}, 3 \mathrm{H}, \mathrm{TBS}), 0.05$ (s, 3H, TBS); ${ }^{13} \mathrm{C} \mathrm{NMR}\left(\mathrm{CDCl}_{3}\right): \delta 175.3,65.9,61.3,59.2,37.6,32.5,25.9,18.2,-5.4$, -5.5; HR MS: calcd for $\mathrm{C}_{12} \mathrm{H}_{27} \mathrm{NO}_{4} \mathrm{SiNa}[\mathrm{M}+\mathrm{Na}]^{+}$300.1602, found 300.1607.

(S)-4-(tert-Butyldimethylsiloxy)-2-hydroxy-N-methoxy-N-methylpentanamide ((S)-5k). HPLC (CHIRALPAK $\mathrm{IC}, i-\mathrm{PrOH} /$ hexane $=1 / 9$, flow rate $=1.0 \mathrm{~mL} / \mathrm{min}): t_{\mathrm{R}}=8.7 \mathrm{~min}(99.6 \%), t_{\mathrm{R}}=21.7 \mathrm{~min}(0.4 \%) ; \mathrm{IR}$ (neat): 3464, $1658 \mathrm{~cm}^{-1} ;{ }^{1} \mathrm{H}$ NMR $\left(\mathrm{CDCl}_{3}\right): \delta 4.47-4.33(\mathrm{~m}, 1 \mathrm{H}, 2-\mathrm{H}), 3.70(\mathrm{~s}, 3 \mathrm{H}, \mathrm{OMe}), 3.64(\mathrm{td}, J=6.0,2.5 \mathrm{~Hz}$, $1 \mathrm{H}, 5-\mathrm{H}), 3.30$ (d, J = 8.0 Hz, 1H, OH), 3.23 (s, 3H, NMe), 1.87-1.78 (m, 1H, 3-H), 1.70-1.51 (m, 3H, 3-H, 4-H), 0.87 (s, 9H, TBS), 0.03 (s, 6H, TBS); ${ }^{13} \mathrm{C} \mathrm{NMR}\left(\mathrm{CDCl}_{3}\right): \delta 175.1,68.5,62.7,61.2,32.4,31.2,28.3,25.9$, 18.3, -5.3; HR MS: calcd for $\mathrm{C}_{13} \mathrm{H}_{29} \mathrm{NO}_{4} \mathrm{SiNa}[\mathrm{M}+\mathrm{Na}]^{+} 314.1758$, found 314.1748.

(R)-2-(Diphenylacetyloxy)-N-methoxy-N-methylpropanamide ((R)-6a). HPLC (CHIRALPAK IC, $i$-PrOH/ hexane $=1 / 4$, flow rate $=1.0 \mathrm{~mL} / \mathrm{min}): t_{\mathrm{R}}=18.9 \mathrm{~min}(26.5 \%), t_{\mathrm{R}}=26.5 \mathrm{in}(96.4 \%)$; IR (neat): 1736, 1673, 1489, 1458, 741, $702 \mathrm{~cm}^{-1} ;{ }^{1} \mathrm{H}$ NMR $\left(\mathrm{CDCl}_{3}\right): \delta 7.36-7.19(\mathrm{~m}, 10 \mathrm{H}, \mathrm{Ph}), 5.39(\mathrm{q}, J=6.8 \mathrm{~Hz}, 1 \mathrm{H}, 2-\mathrm{H}), 5.13$ (s, 1H, 2'-H), 3.73 (s, 3H, OMe), $3.18(\mathrm{~s}, 3 \mathrm{H}, \mathrm{NMe}), 1.41(\mathrm{~d}, J=6.8 \mathrm{~Hz}, 3 \mathrm{H}, 3-\mathrm{H}) ;{ }^{13} \mathrm{C} \mathrm{NMR}\left(\mathrm{CDCl}_{3}\right): \delta$ $172.5,170.6,138.6,138.5,128.8,128.7,128.6,128.4,127.2,127.1,68.3,56.6,32.1,16.3$; HR MS: calcd for $\mathrm{C}_{19} \mathrm{H}_{21} \mathrm{NO}_{4} \mathrm{Na}[\mathrm{M}+\mathrm{Na}]^{+} 350.1363$, found 350.1350 .

(R)-2-(Diphenylacetyloxy)-N-methoxy-N-methylbutanamide ((R)-6b). HPLC (CHIRALPAK IC, $i$-PrOH/ hexane $=1 / 4$, flow rate $=1.0 \mathrm{~mL} / \mathrm{min}): t_{\mathrm{R}}=13.6 \mathrm{~min}(2.2 \%), t_{\mathrm{R}}=30.8 \mathrm{~min}(97.8 \%) ; \mathrm{IR}$ (neat): 1736, 1676, 1486, 1454, 749, $699 \mathrm{~cm}^{-1} ;{ }^{1} \mathrm{H}$ NMR $\left(\mathrm{CDCl}_{3}\right): \delta 7.39-7.19(\mathrm{~m}, 10 \mathrm{H}, \mathrm{Ph}), 5.25(\mathrm{t}, J=7.0 \mathrm{~Hz}, 1 \mathrm{H}, 2-\mathrm{H})$, $5.16\left(\mathrm{~s}, 1 \mathrm{H}, 2^{\prime}-\mathrm{H}\right), 3.76(\mathrm{~s}, 3 \mathrm{H}, \mathrm{OMe}), 3.20$ (s, 3H, NMe), 1.85-1.76 (m, 2H, 3-H), $0.88(\mathrm{t}, J=7.0 \mathrm{~Hz}, 3 \mathrm{H}$, $4-\mathrm{H}) ;{ }^{13} \mathrm{C} \mathrm{NMR}\left(\mathrm{CDCl}_{3}\right): \delta 172.7,170.0,138.6,138.5,128.8,128.8,128.6,128.4,127.2,127.1,73.0,61.2$, 56.7, 32.0, 24.1, 9.7; HR MS: calcd for $\mathrm{C}_{20} \mathrm{H}_{23} \mathrm{NO}_{4} \mathrm{Na}[\mathrm{M}+\mathrm{Na}]^{+} 364.1519$, found 364.1537. 
(R)-2-(Diphenylacetyloxy)-N-methoxy-N-methylpentanamide ((R)-6c). HPLC (CHIRALPAK IC, $i$-PrOH/ hexane $=1 / 9$, flow rate $=1.0 \mathrm{~mL} / \mathrm{min}): t_{\mathrm{R}}=13.2 \mathrm{~min}(1.9 \%), t_{\mathrm{R}}=32.7 \mathrm{~min}(98.1 \%) ; \mathrm{IR}($ neat $): 1736$, $1678,1602,1497,1459,740,698 \mathrm{~cm}^{-1} ;{ }^{1} \mathrm{H} \mathrm{NMR}\left(\mathrm{CDCl}_{3}\right): \delta 7.35-7.16(\mathrm{~m}, 10 \mathrm{H}, \mathrm{Ph}), 5.28(\mathrm{dd}, J=9.0,3.5$ $\mathrm{Hz}, 1 \mathrm{H}, 2-\mathrm{H}), 5.12$ (s, 1H, 2'-H), 3.73 (s, 3H, OMe), 3.16 (s, 3H, NMe), 1.80-1.61 (m, 2H, 3-H), $1.39-1.16$ $(\mathrm{m}, 2 \mathrm{H}, 4-\mathrm{H}), 0.81(\mathrm{dd}, J=7.5,7.5 \mathrm{~Hz}, 3 \mathrm{H}, 5-\mathrm{H}) ;{ }^{13} \mathrm{C} \mathrm{NMR}\left(\mathrm{CDCl}_{3}\right): \delta 172.7,170.2,138.6,138.5,128.8$, $128.8,128.5,128.4,127.2,127.1,71.6,61.2,56.7,32.6,32.1,18.5,13.4 ;$ HR MS: calcd for $\mathrm{C}_{21} \mathrm{H}_{25} \mathrm{NO}_{4} \mathrm{Na}[\mathrm{M}$ $+\mathrm{Na}]^{+} 378.1676$, found 378.1689 .

(R)-2-(Diphenylacetyloxy)-N-methoxy-N,3-dimethylbutanamide ((R)-6d). HPLC (CHIRALPAK IC, $i-\mathrm{PrOH} /$ hexane $=1 / 9$, flow rate $=1.0 \mathrm{~mL} / \mathrm{min}): t_{\mathrm{R}}=11.3 \mathrm{~min}(17.1 \%), t_{\mathrm{R}}=32.5 \mathrm{~min}(82.9 \%) ; \mathrm{IR}$ (neat): 1735, 1674, 1496, 750, $700 \mathrm{~cm}^{-1},{ }^{1} \mathrm{H} \mathrm{NMR}\left(\mathrm{CDCl}_{3}\right): \delta 7.42-7.21(\mathrm{~m}, 10 \mathrm{H}, \mathrm{Ph}), 5.17\left(\mathrm{~s}, 1 \mathrm{H}, 2^{\prime}-\mathrm{H}\right)$, $5.17(\mathrm{~d}, J=6.5 \mathrm{~Hz}, 1 \mathrm{H}, 2-\mathrm{H}), 3.79$ (s, 3H, OMe), 3.22 (s, 3H, NMe), 2.17 (dqq, J = 7.0, 6.5, 6.5 Hz, 1H, $3-\mathrm{H}), 0.88(\mathrm{~d}, J=6.5 \mathrm{~Hz}, 3 \mathrm{H}, 4-\mathrm{H}), 0.87(\mathrm{~d}, J=6.5 \mathrm{~Hz}, 3 \mathrm{H}, 4-\mathrm{H}) ;{ }^{13} \mathrm{C} \mathrm{NMR}\left(\mathrm{CDCl}_{3}\right): \delta 172.6,169.5,138.6$, 138.5, 128.8, 128.8, 128.6, 128.3, 127.2, 127.0, 75.8, 61.1, 56.9, 32.0, 29.9, 18.7, 17.3; HR MS: calcd for $\mathrm{C}_{21} \mathrm{H}_{25} \mathrm{NO}_{4} \mathrm{Na}[\mathrm{M}+\mathrm{Na}]^{+} 378.1676$, found 378.1686 .

(R)-2-(Diphenylacetyloxy)-N-methoxy-N-methylhexanamide ((R)-6e). HPLC (CHIRALPAK IC, $i$-PrOH/ hexane $=1 / 9$, flow rate $=1.0 \mathrm{~mL} / \mathrm{min}): t_{\mathrm{R}}=10.4 \mathrm{~min}(1.8 \%), t_{\mathrm{R}}=25.4 \mathrm{~min}(98.2 \%) ; \mathrm{IR}($ neat $): 1736,1678$, 1498, 1445, 743, $704 \mathrm{~cm}^{-1} ;{ }^{1} \mathrm{H}$ NMR $\left(\mathrm{CDCl}_{3}\right): \delta 7.35-7.17(\mathrm{~m}, 10 \mathrm{H}, \mathrm{Ph}), 5.27(\mathrm{dd}, J=8.5,4.0 \mathrm{~Hz}, 1 \mathrm{H}, 2-\mathrm{H})$, 5.13 (s, 1H, 2-H), 3.73 (s, 3H, OMe), 3.16 (s, 3H, NMe), 1.80-1.67 (m, 2H, 3-H), 1.29-1.13 (m, 4H, 4-H, 5-H), 0.79 (ddd, $J=7.0,7.0,2.5 \mathrm{~Hz}, 3 \mathrm{H}, 6-\mathrm{H}) ;{ }^{13} \mathrm{C} \mathrm{NMR}\left(\mathrm{CDCl}_{3}\right): \delta 172.6,170.2,138.6,138.5,128.8,128.8$, 128.6, 128.4, 127.2, 127.1, 71.7, 61.2, 56.7, 21.1, 30.2, 27.2, 22.0, 13.7; HR MS: calcd for $\mathrm{C}_{22} \mathrm{H}_{27} \mathrm{NO}_{4} \mathrm{Na}[\mathrm{M}$ $+\mathrm{Na}]^{+} 392.1832$, found 392.1848 .

(R)-2-(Diphenylacetyloxy)-N-methoxy-N,4-dimethylpentanamide ((R)-6f). HPLC (CHIRALPAK IC, $i$-PrOH/ hexane $=1 / 9$, flow rate $=1.0 \mathrm{~mL} / \mathrm{min}): t_{\mathrm{R}}=10.1 \mathrm{~min}(1.8 \%), t_{\mathrm{R}}=24.7 \mathrm{~min}(98.2 \%) ; \mathrm{IR}$ (neat): 1733, 1678, 1491, 752, $702 \mathrm{~cm}^{-1} ;{ }^{1} \mathrm{H}$ NMR $\left(\mathrm{CDCl}_{3}\right): \delta 7.34-7.17(\mathrm{~m}, 10 \mathrm{H}, \mathrm{Ph}), 5.31(\mathrm{dd}, J=10.3,3.0 \mathrm{~Hz}, 1 \mathrm{H}$, 2-H), 5.12 (s, 1H, 2'-H), 3.74 (s, 3H, OMe), 3.15 (s, 3H, NMe), 1.73 (ddd, J = 14.0, 10.0, 4.0 Hz, 1H, 3-H), 1.58-1.48 (m, 1H, 4-H), $1.45(\mathrm{ddd}, J=14.0,9.5,3.5 \mathrm{~Hz}, 1 \mathrm{H}, 3-\mathrm{H}), 0.80(\mathrm{~d}, J=6.0 \mathrm{~Hz}, 3 \mathrm{H}, 5-\mathrm{H}), 0.76$ (d, $J$ $=6.5 \mathrm{~Hz}, 5-\mathrm{H}) ;{ }^{13} \mathrm{C} \mathrm{NMR}\left(\mathrm{CDCl}_{3}\right): \delta 172.7,170.6,138.5,138.5,128.8,128.8,128.6,128.4,127.2,127.1$, 70.6, 61.2, 56.7, 39.2, 32.2, 24.5, 23.1, 21.1; HR MS: calcd for $\mathrm{C}_{22} \mathrm{H}_{27} \mathrm{NO}_{4} \mathrm{Na}[\mathrm{M}+\mathrm{Na}]^{+}$392.1832, found 392.1847 .

(R)-2-Cyclohexyl-2-(diphenylacetyloxy)-N-methoxy-N-methylacetamide ((R)-6g). HPLC (CHIRALPAK IC, $i-\mathrm{PrOH} /$ hexane $=1 / 9$, flow rate $=1.0 \mathrm{~mL} / \mathrm{min}): t_{\mathrm{R}}=10.3 \mathrm{~min}(20.4 \%), t_{\mathrm{R}}=40.0 \mathrm{~min}(79.6 \%) ; \mathrm{IR}$ (neat): 1736, 1672, 1495, 1451, 752, $700 \mathrm{~cm}^{-1}{ }^{1} \mathrm{H} \mathrm{NMR}\left(\mathrm{CDCl}_{3}\right): \delta 7.35-7.16(\mathrm{~m}, 10 \mathrm{H}, \mathrm{Ph}), 5.13(\mathrm{~d}, J=6.5 \mathrm{~Hz}$, $1 \mathrm{H}, 2-\mathrm{H}), 5.11$ (s, 1H, 2'-H), 3.75 (s, 3H, OMe), 3.17 (s, 3H, NMe), 1.84-1.75 (m, 1H, c-Hex), 1.68-1.41 (m, 5H, c-Hex), 1.24-0.92 (m, 5H, c-Hex); ${ }^{13} \mathrm{C} \mathrm{NMR}\left(\mathrm{CDCl}_{3}\right): \delta 172.6,169.5,138.6,138.5,128.8,128.6,128.3$, $127.2,127.0,75.4,61.1,56.9,39.3,31.9,28.7,27.8,26.0,25.7$; HR MS: calcd for $\mathrm{C}_{24} \mathrm{H}_{29} \mathrm{NO}_{4} \mathrm{Na}[\mathrm{M}+\mathrm{Na}]^{+}$ 418.1989, found 418.2003.

(R)-3-(tert-Butyldimethylsiloxy)-2-(diphenylacetyloxy)-N-methoxy-N-methylpropanamide ((R)-6i). HPLC $($ CHIRALPAK IC, $i$-PrOH/hexane $=1 / 9$, flow rate $=0.5 \mathrm{~mL} / \mathrm{min}): t_{\mathrm{R}}=13.7 \mathrm{~min}(93.0 \%), t_{\mathrm{R}}=21.2 \mathrm{~min}$

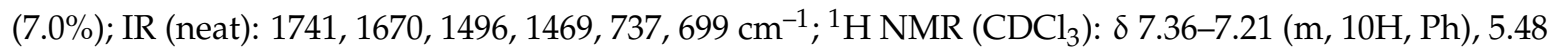
$(\mathrm{dd}, J=7.0,4.0 \mathrm{~Hz}, 1 \mathrm{H}, 2-\mathrm{H}), 5.17\left(\mathrm{~s}, 1 \mathrm{H}, 2^{\prime}-\mathrm{H}\right), 3.91(\mathrm{dd}, J=11.0,4.0 \mathrm{~Hz}, 1 \mathrm{H}, 3-\mathrm{H}), 3.88(\mathrm{dd}, J=11.0,7.0$ Hz, 1H, 3-H), 3.80 (s, 3H, OMe), 3.20 (s, 3H, NMe), 0.83 (s, 9H, TBS), -0.00 (s, 3H, TBS), -0.03 (s, 3H, $\mathrm{TBS}) ;{ }^{13} \mathrm{C} \mathrm{NMR}\left(\mathrm{CDCl}_{3}\right): \delta$ 172.6, 167.7, 138.5, 138.4, 128.8, 128.8, 128.6, 128.4, 127.2, 127.1, 73.3, 62.0, 61.3, 56.7, 32.1, 25.7, 18.2, -5.5, -5.6; HR MS: calcd for $\mathrm{C}_{25} \mathrm{H}_{35} \mathrm{NO}_{5} \mathrm{SiNa}[\mathrm{M}+\mathrm{Na}]^{+} 480.2177$, found 480.2174 . 
(R)-4-(tert-Butyldimethylsiloxy)-2-(diphenylacetyloxy)-N-methoxy-N-methylbutanamide ((R)-6j). HPLC $($ CHIRALPAK IC, $i$-PrOH $/$ hexane $=1 / 9$, flow rate $=1.0 \mathrm{~mL} / \mathrm{min}): t_{\mathrm{R}}=9.8 \mathrm{~min}(2.7 \%), t_{\mathrm{R}}=23.4$ $\min (97.3 \%)$; IR (neat): 1738, 1673, 1496, 1469, 762, $701 \mathrm{~cm}^{-1} ;{ }^{1} \mathrm{H}$ NMR $\left(\mathrm{CDCl}_{3}\right): \delta 7.38-7.21(\mathrm{~m}, 10 \mathrm{H}$, $\mathrm{Ph}), 5.25$ (d, J = 8.5 Hz, 1H, 2-H), 5.16 (s, 1H, 2'-H), 3.78 (s, 3H, OMe), 3.59 (ddd, J = 10.0, 6.0, 4.0 Hz, $1 \mathrm{H}, 4-\mathrm{H}), 3.50$ (ddd, 10.0, 10.0, $\left.5.0 \mathrm{~Hz}, 1 \mathrm{H}, 4^{\prime}-\mathrm{H}\right), 3.21$ (s, 3H, NMe), 2.04-1.84 (m, 2H, 3-H), 0.85 (s, 9H, TBS), -0.03 (s, 3H, TBS), -0.07 (s, 3H, TBS); ${ }^{13} \mathrm{C} \mathrm{NMR}\left(\mathrm{CDCl}_{3}\right): \delta 172.5,170.4,138.6,138.5,128.8$, 128.8, 128.6, 128.4, 127.2, 127.1, 68.7, 61.2, 58.3, 56.8, 33.6, 32.2, 25.8, 18.1, -5.5, -5.6; HR MS: calcd for $\mathrm{C}_{26} \mathrm{H}_{37} \mathrm{NO}_{5} \mathrm{SiNa}\left(\mathrm{M}+\mathrm{Na}^{+}\right) 494.2333$, found 494.2321 .

(R)-4-(tert-Butyldimethylsiloxy)-2-(diphenylacetyloxy)-N-methoxy-N-methylpentanamide ((R)-6k). HPLC $($ CHIRALPAK IC, $i$-PrOH $/$ hexane $=1 / 9$, flow rate $=1.0 \mathrm{~mL} / \mathrm{min}): t_{\mathrm{R}}=8.8 \mathrm{~min}(3.0 \%), t_{\mathrm{R}}=21.4 \mathrm{~min}$ (97.0\%); IR (neat): 1739, 1680, 1496, 1469, 735, $701 \mathrm{~cm}^{-1} ;{ }^{1} \mathrm{H} \mathrm{NMR}\left(\mathrm{CDCl}_{3}\right): \delta 7.39-7.20(\mathrm{~m}, 10 \mathrm{H}, \mathrm{Ph})$, $5.34(\mathrm{dd}, J=8.5,4.5 \mathrm{~Hz}, 1 \mathrm{H}, 2-\mathrm{H}), 5.16\left(\mathrm{~s}, 1 \mathrm{H}, 2^{\prime}-\mathrm{H}\right), 3.77$ (s, 3H, OMe), 3.54 (t, J = 6.0 Hz, 2H, 5-H), 3.20 (s, 3H, NMe), 1.93-1.77 (m, 2H, 3-H), 1.58-1.42 (m, 2H, 4-H), 0.87 (s, 9H, TBS), 0.01 (s, 6H, TBS); ${ }^{13} \mathrm{C}$ NMR $\left(\mathrm{CDCl}_{3}\right): \delta 172.6,170.1,138.6,138.5,128.8,128.8,128.6,128.4,127.2,127.1,71.8,62.3,61.2,56.7$, 32.1, 28.4, 27.2, 25.9, 18.2, -5.4; HR MS: calcd for $\mathrm{C}_{27} \mathrm{H}_{39} \mathrm{NO}_{5} \mathrm{SiNa}[\mathrm{M}+\mathrm{Na}]^{+}$508.2490, found 508.2514.

The ${ }^{1} \mathrm{H}$ and ${ }^{13} \mathrm{C}-\mathrm{NMR}$ spectra of the compounds are available in Supplementary Materials.

\section{Conclusions}

In summary, we developed an efficient method for producing optically active 2-hydroxyamides based on the $\mathrm{KR}$ of racemic 2-hydroxyamides with diphenylacetyl components using $(R)$-BTM as a nucleophilic chiral acyl-transfer catalyst. The resulting chiral compounds could be converted into the other useful chiral compounds without erosion of the chirality. The transition states were determined by DFT calculations to support the observations in their process. Further research on the present method, including the application of this novel protocol to the production of other chiral materials, is currently underway in our laboratory.

Supplementary Materials: The Supplementary Materials containing $1 \mathrm{H}$ and 13C NMR spectroscopic data are available online, Figure S1: Preferable transition structure ((R)-3a-TS), Table S1: Cartesian Coordinates (Angstroms), Figure S2: Unfavorable transition structure ((S)-3a-TS), Table S2: Cartesian Coordinates (Angstroms), Figure S3: Preferable transition structure ((R)-5a-TS), Table S3: Cartesian Coordinates (Angstroms), Figure S4: Unfavorable transition structure ((S)-5a-TS), Table S4: Cartesian Coordinates (Angstroms).

Author Contributions: K.N. and I.S. conceived and designed the experiments; T.M., T.K., A.S., R.I., K.O., K.N. and I.S. carried out the synthesis and characterization of all compounds; All authors discussed the results of the experiments.

Funding: This research was funded by Grants-in-Aid for Scientific Research from the Ministry of Education, Science, Sports and Culture, Japan.

Conflicts of Interest: The authors declare no conflict of interest.

\section{References}

1. Ahmand, S.; Ashfaq, A.; Alam, M.; Bisacchi, G.S.; Chen, P.C.; Cheng, P.T.W.; Greytok, J.A.; Hermsmeier, M.A.; Lin, P.-F.; Lis, K.A.; et al. $\alpha$-Hydroxyamide derived aminodiols as potent inhibitors of HIV protease. Bioorg. Med. Chem. Lett. 1995, 5, 1729-1734. [CrossRef]

2. Punniyamurthy, T.; Iqbal, J. Polyanilone supported cobalt(II) salen catalysed synthesis of pyrrolidine containing $\alpha$-hydroxyamide core structures as inhibitors for HIV proteases. Tetrahedron Lett. 1997, 38, 4463-4466. [CrossRef]

3. Jones, P.J.; Wang, Y.; Smith, M.D.; Hargus, N.J.; Eidam, H.S.; White, H.S.; Kapur, J.; Brown, M.L.; Patel, M.K. Hydroxyamide analogs of propofol exhibit state-dependent block of sodium channels in hippocampal neurons: Implications for anticonvulsant activity. J. Pharm. Exp. Ther. 2007, 320, 828-836. [CrossRef] [PubMed] 
4. Xu, G.G.; Etzkorn, F.A. Convergent synthesis of $\alpha$-ketoamide inhibitors of Pin1. Org. Lett. 2010, 12, 696-699. [CrossRef] [PubMed]

5. Sánchez-Carnerero, E.M.M.; Engel, T.D.L.C.; Maroto, B.; Cerero, S.D.L.M. Unexpected efficiency of non- $C_{2}$-symmetric bis(hydroxyamide)-based zinc-chelate catalysts. Chirality 2011, 23, 523-526. [CrossRef] [PubMed]

6. Geoghegan, P.; O'Leary, P. Hydroxyamide-based ligands and their use in the asymmetric catalysis of key organic transformations. ACS Catal. 2012, 2, 573-591. [CrossRef]

7. Szymanski, W.; Ostaszewski, R. Chemoenzymatic synthesis of enantiomerically enriched $\alpha$-hydroxyamides. J. Mol. Catal. B Enzym. 2007, 47, 125-128. [CrossRef]

8. Pasquier, C.; Naili, S.; Pelinski, L.; Brocard, J.; Mortreux, A.; Agbossou, F. Synthesis and application in enantioselective hydrogenation of new free and chromium complexed aminophosphine-phosphinite ligands. Tetrahedron Asymmetry 1998, 9, 193-196. [CrossRef]

9. Evans, D.A.; Aye, Y.; Wu, J. Asymmetric, anti-selective scandium-catalyzed Sakurai additions to glyoxyamide. Applications to the synthesis of N-boc D-alloisoleucine and D-isoleucine. Org. Lett. 2006, 8, 2071-2073. [CrossRef] [PubMed]

10. Bayat, M.; Nasri, S.; Hosseini, H.; Hassanzadeh, F. One-pot synthesis of $\alpha$-hydroxyamides using alkyl isocyanides and aryl aldehydes. Monatsh. Chem. 2012, 143, 801-804. [CrossRef]

11. Vedejs, E.; Jure, M. Efficiency in nonenzymatic kinetic resolution. Angew. Chem. Int. Ed. 2005, 44, 3974-4001. [CrossRef] [PubMed]

12. Müller, C.E.; Schreiner, P.R. Organocatalytic enantioselective acyl transfer onto racemic as well as meso alcohols, amines, thiols. Angew. Chem. Int. Ed. 2011, 50, 6012-6042. [CrossRef] [PubMed]

13. Pellissier, H. Catalytic non-enzymatic kinetic resolution. Adv. Synth. Catal. 2011, 353, 1613-1666. [CrossRef]

14. Shiina, I.; Nakata, K. The first asymmetric esterification of free carboxylic acids with racemic alcohols using benzoic anhydrides and tetramisole derivatives: An application to the kinetic resolution of secondary benzylic alcohols. Tetrahedron Lett. 2007, 48, 8314-8317. [CrossRef]

15. Shiina, I.; Nakata, K.; Sugimoto, M.; Onda, Y.; Iizumi, T.; Ono, K. 2,2-Disubstituted propionic anhydrides: Effective coupling reagents for the kinetic resolution of secondary benzylic alcohols using BTM. Heterocycles 2009, 77, 801-810. [CrossRef]

16. Nakata, K.; Shiina, I. An effective kinetic resolution of racemic secondary benzylic alcohols using 3-pyridinecarboxylic anhydride and a chiral acyl-transfer catalsyt in the absence of tertiary amine. Heterocycles 2010, 80, 169-175.

17. Shiina, I.; Ono, K.; Nakata, K. Kinetic resolution of racemic 1-heteroarylalkanols by asymmetric esterification using diphenylacetic acid with pivalic anhydride and achiral acyl-transfer catalyst. Chem. Lett. 2011, 40, 147-149. [CrossRef]

18. Nakata, K.; Ono, K.; Shiina, I. Kinetic resolution of the racemic 1-(aryloxazol)carbinols with ahiral carboxylic acids by asymmetric esterification: A new method for the preparation of chiral 1,2-amino alcohols. Heterocycles 2011, 82, 1171-1180.

19. Nakata, K.; Shiina, I. (R)-(+)-N-Methylbenzoguanidine ((R)-NMBG) catalyzed kinetic resolution of racemic secondary benzylic alcohols with free carboxylic acids by asymmetric esterification. Org. Biomol. Chem. 2011, 9, 7092-7096. [CrossRef] [PubMed]

20. Shiina, I.; Nakata, K.; Ono, K.; Mukaiyama, T. Kinetic resolution of racemic secondary benzylic alcohols by the enantioselective esterification using pyridine-3-carboxylic anhydride (3-PCA) with chiral acyl-transfer catalysts. Helv. Chim. Acta 2012, 95, 1891-1911. [CrossRef]

21. Shiina, I.; Nakata, K.; Onda, Y. Kinetic resolution of racemic carboxylic acids using achiral alcohols by the promotion of benzoic anhydrides and tetramisole derivatives: Production of chiral nonsteroidal anti-inflammatory drugs and their esters. Eur. J. Org. Chem. 2008, 5887-5890. [CrossRef]

22. Nakata, K.; Onda, Y.; Ono, K.; Shiina, I. An effective kinetic resolution of racemic $\alpha$-arylpronanoic acids, $\alpha$-arylbutanoic acids, and $\beta$-substituted- $\alpha$-arylpropanoic acids with bis(9-phenanthryl)methanol as a new achiral nucleophile in the asymmetric esterification using carboxylic anhydrides and the acyl-transfer catalyst. Tetrahedron Lett. 2010, 51, 5666-5669.

23. Shiina, I.; Nakata, K.; Ono, K.; Onda, Y.; Itagaki, M. Kinetic resolution of racemic $\alpha$-arylalkanoic acids with achiral alcohols via the asymmetric esterification using carboxylic anhydrides and acyl-transfer catalysts. J. Am. Chem. Soc. 2010, 132, 11629-11641. [CrossRef] [PubMed] 
24. Shiina, I.; Ono, K.; Nakata, K. Non-enzymatic dynamic kinetic resolution of racemic $\alpha$-arylalkanoic acids: An advanced asymmetric synthesis of chiral nonsteroidal anti-inflammatory drugs (NSAIDs). Catal. Sci. Technol. 2012, 2, 2200-2205. [CrossRef]

25. Tengeiji, A.; Shiina, I. A new method for production of chiral 2-aryl-2-fluoropropanoic acids using an effective kinetic resolution of racemic 2-aryl-2-fluoropropanoic acids. Molecules 2012, 17, 7356-7378. [CrossRef] [PubMed]

26. Tengeiji, A.; Nakata, K.; Ono, K.; Shiina, I. A new method for production of chiral 2-aryloxypropanoic acids using effective kinetic resolution of racemic 2-aryloxycarboxylic acids. Heterocycles 2012, 86, 1227-1252.

27. Shiina, I.; Nakata, K.; Ono, K.; Sugimoto, M.; Sekiguchi, A. Kinetic resolution of racemic 2-hydroxyalkanoates using the enantioselective mixed-anhydride method with pivalic anhydride and a chiral acyl-transfer catalyst. Chem. Eur. J. 2010, 16, 167-172. [CrossRef] [PubMed]

28. Nakata, K.; Gotoh, K.; Ono, K.; Futami, K.; Shiina, I. Kinetic resolution of racemic 2-hydroxy- $\gamma$-butyrolactones by asymmetric esterification using diphenylacetic acid with pivalic anhydride and a chiral acyl-transfer catalyst. Org. Lett. 2013, 15, 1170-1173. [CrossRef] [PubMed]

29. Nakata, K.; Sekiguchi, A.; Shiina, I. A convenient method for the kinetic resolution of racemic 2-hydroxyalkanoates using diphenylacetic anhydride (DPHAA) and a chiral acyl-transfer catalyst. Tetrahedron Asymmetry 2011, 22, 1610-1619. [CrossRef]

30. Kagan, H.B.; Fiaud, J.C. Kinetic resolution. Top. Stereochem. 1988, 18, 249-330.

31. Nahm, S.; Weinreb, S. N-Methoxy- $n$-methylamides as effective acylating agents. Tetrahedron Lett. 1981, 22, 3815-3818. [CrossRef]

32. Khlesthin, V.K.; Mazhukin, D.G. Recent advances in the application of N,O-dialkylhydroxyamines in organic chemistry. Curr. Org. Chem. 2003, 7, 967-993. [CrossRef]

33. Sivaraman, B.; Singh, A.I. The growing synthetic utility of the new Weinreb amide. Synthesis 2008, 23, 3707-3738.

34. All Calculations Were Performed with the Program Package Spartan '10 1.1.0 of Wavefunction Inc. Available online: http:/ / www.wavefun.com (accessed on 8 August 2018).

Sample Availability: Available.

(C) 2018 by the authors. Licensee MDPI, Basel, Switzerland. This article is an open access article distributed under the terms and conditions of the Creative Commons Attribution (CC BY) license (http:/ / creativecommons.org/licenses/by/4.0/). 